\title{
Development of General Purpose Data Acquisition Shell (GPDAS)
}

\author{
Y. Chung and K. Kim
}

Advanced Photon Source

Argonne National Laboratory

Argonne, IL 60439

\section{DISCLAIMER}

This report was prepared as an account of work sponsored by an agency of the United States Government. Neither the United States Government nor any agency thereof, nor any of their employees, makes any warranty, express or implied, or assumes any legal liability or responsibility for the accuracy, completeness, or usefulness of any information, apparatus, product, or process disclosed, or represents that its use would not infringe privately owned rights. Reference herein to any specific commercial product, process, or service by trade name, trademark, manufacturer, or otherwise does not necessarily constitute or imply its endorsement, recommendation, or favoring by the United States Government or any agency thereof. The views and opinions of authors expressed herein do not necessarily state or reflect those of the United States Government or any agency thereof. 


\section{DISCLAIMER}

Portions of this document may be illegible in electronic image products. Images are produced from the best available original document. 


\section{Table of Contents}

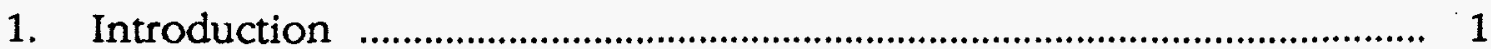

2. Configuration ................................................................................ 2

3. Starting and Programming in GPDAS _................................................. 2

4. Constants and Variables ...................................................................... 4

5. System Global Variables ……………..................................................... 6

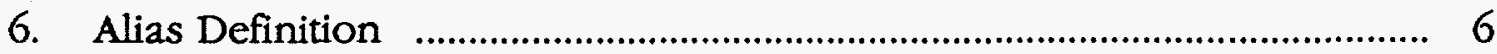

7. History Mechanism .................................................................................. 7

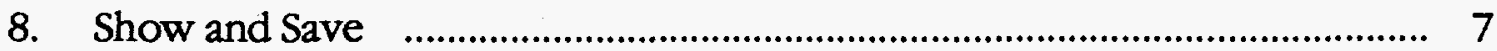

9. Input and Output

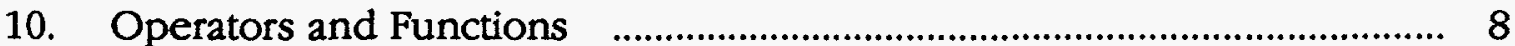

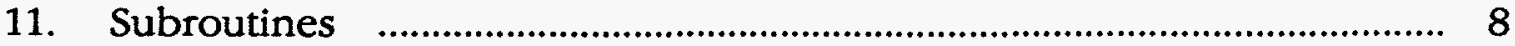

12. Command Flow Control _.................................................................... 9

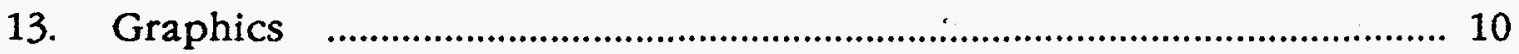

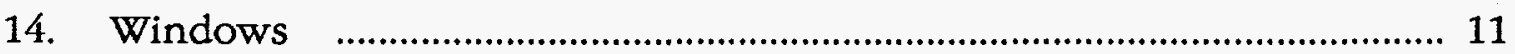

15. GPIB and Device Drivers

16. Help Facility ......................................................................................... 12

\section{Appendices}

A. GPDAS.CFG (GPDAS Configuration File) ……..................................... 14

B. GPDAS Shell Command Syntax ………............................................ 16

C. Special Characters and System Global Variables ………........................... 21

D. I/O Command Syntax .................................................................... 22

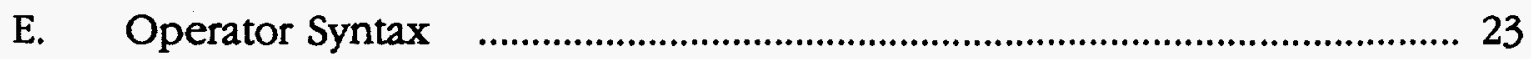

F. Graphics Command Syntax ……......................................................... 26

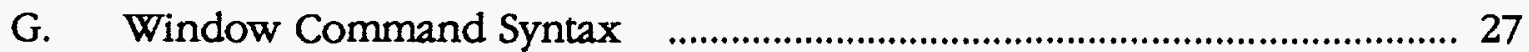

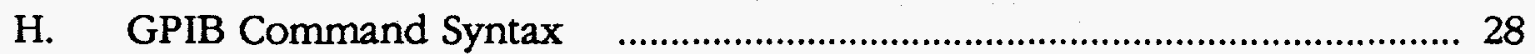

I. Device Driver Command Syntax

J. Script Example SORTCAP.SCR …………....................................... 32

K. Script Example RADTRIG.SCR _...................................................... 41 


\section{Introduction}

GPDAS (General Purpose Data Acquisition Shell) has grown out of a need for a command interpreter and a programming environment designed to handle streamlined data acquisition and analysis in a flexible laboratory measurement setting. Although most operating systems on various platforms provide a command interpreter and certain levels of programmability, there are none specifically designed for a laboratory environment dedicated to data acquisition, analysis, and device control that requires command flow control, communication with external devices, and storage and archiving of acquired data.

Usually a dedicated, stand-alone application, though limited in its scope of capabilities, would be developed for such purposes. The drawback of this approach, however, is that such applications provide little flexibility to adapt to different laboratory environments or different configurations of devices, especially in the R\&D phase which calls for frequent changes in the measurement procedures.

An interpreter-based shell can be a very convenient tool for device control and data acquisition through the user command input as well as via script files containing several commands. By incorporating the native operating system, such as UNIX or MSDOS, it can take advantage of existing utility programs for file manipulation and data analysis. Hardware-level debugging and script programming can also be facilitated by utilizing interactive mode, aliases, variables, and history mechanisms. In consideration of the interpreter's relatively slow execution time, some routines requiring high speed can be separated as module programs, or utilities, and called from scripts. Modularization of an application into several small utilities is also a good programming strategy since they are easy to debug, don't take up much memory space, and can be used in other contexts.

The current version of GPDAS runs on MS-DOS-based microcomputers. The software package consists of a shell, utilities, and script programs. The computer system for magnet measurement at APS, for instance, currently uses GPDAS and consists of a Compaq 386/20e microcomputer equipped with 4 MB RAM, a $40 \mathrm{MB}$ hard drive, a $44 \mathrm{MB}$ removable hard drive, a 5.25" $1.2 \mathrm{MB}$ floppy drive, a 3.5" $1.44 \mathrm{MB}$ floppy drive, and a VGA monitor. The system is linked to other computers through Ethernet for data transfer and communication. Text and PostScript graphics files are downloaded to laser printers using an AppleTalk connection. 
The shell can be directly interfaced with PC boards in order to control the data acquisition devices. In conjunction with the magnet measurement system, software drivers for IEEE-488, a multifunction board, and an ADC/DAC are built into the shell. The shell can be interfaced to other types of boards through source level modification. Developed with such possibilities in mind, the internal structure of the shell is highly modularized to facilitate such changes.

This note is intended as an abbreviated introduction to the concept and the structure of GPDAS and assumes the reader has a certain level of familiarity with programming in general. The structure of the following sections consists of brief explanations of the concepts and commands of GPDAS, followed by several examples. Some of these are tabulated in the appendices at the end of this note.

\section{Configuration}

When started, GPDAS reads in several setup parameters from the configuration file. The default configuration file has the same name and is in the same directory as the shell application, with the file extension ".CFG". The syntax of this file and the meaning of each parameter are shown in Appendix A. Default values are used for unspecified parameters. If the file cannot be located in the present working directory, default values are used for the parameters that are essential in starting up the shell. The configuration parameters for the device drivers, e.g., GPIB and ATMIO, are read in the first time those drivers are called.

\section{Starting and Programming in GPDAS}

The shell is started by typing in the name of the shell program with extension ".EXE" at the DOS prompt. It takes the configuration file name as the only optional argument preceded by "-h".

Ex. 3.1: Starting up the GPDAS shell at DOS prompt.

$$
\begin{aligned}
& C: \backslash>\mathrm{mm} \quad \text { ! name of the shell program is mm.exe and the } \\
& \text { ! configuration file name is } \mathrm{mm} . \mathrm{cfg} \text {. } \\
& c: \backslash>\text { diag -h gpdas.cfg ! name of the shell program is } \\
& \text { diag.exe and the configuration } \\
& \text { file name is gpdas.cfg. }
\end{aligned}
$$


The shell can be programmed to perform desired tasks either interactively or non-interactively. In the interactive mode commands are entered through the keyboard, while in non-interactive mode the shell reads commands from a script and executes them. The user interface of the interactive mode is very similar to that of the native operating system, MS-DOS.

The syntax of the commands must conform to DASL (Data Acquisition Shell Language), a high-level programming language developed for GPDAS. Commands not understood by the shell are passed to MS-DOS for further processing. These shell commands are listed in Appendix B. Script programming is easier than developing new applications; therefore, debugging of hardware and testing of measurement procedures are simplified.

Ex. 3.2: Sample commands at the shell prompt level.

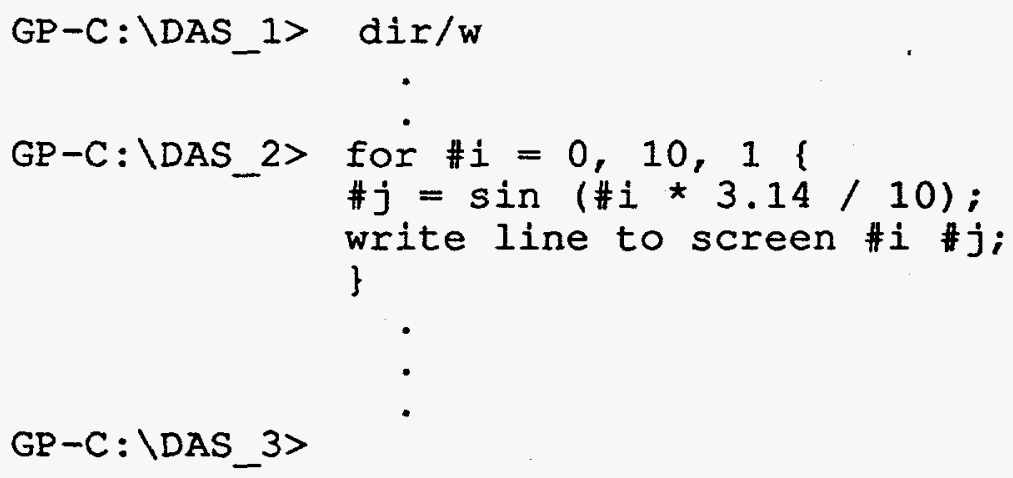

A script should begin with the "procedure" command followed by the script name and a left curly bracket. This signifies the beginning of the script. The left curly bracket must be matched by a right bracket at the end of the script, which is the implicit end of the script. The "end" command explicitly terminates the script execution. Each command in a script must end with a semicolon (;). A line that begins with an exclamation mark (!) is a comment line and is ignored. If a line starts with an exclamation mark and an asterisk (!*), all the following characters up to "*!" are ignored. An example script is shown in Ex. 3.3.

Ex 3.3: A sample script.

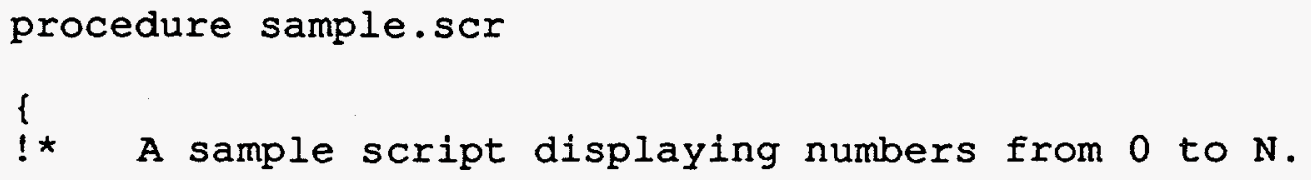




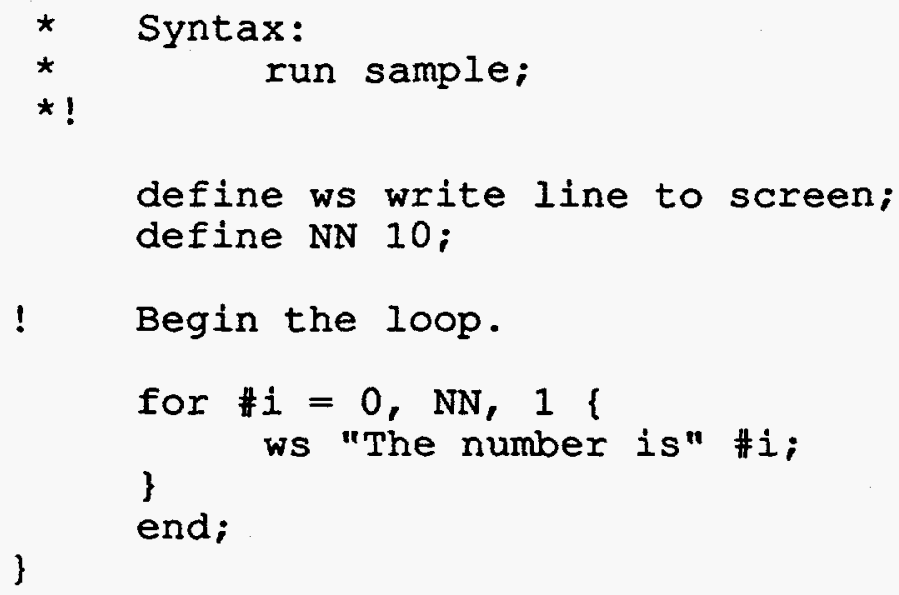

Design objectives of the shell include:

- Interactive mode for user command input

- Non-interactive mode for script execution

- Definition of command aliases

- History of commands

- Assignment of variables and arrays (integer, long, float, double, and string) in RAM and virtual memory

- Backup of aliases, history, and variables in script format

- Mathematical, logical, and text string operations

- Input and output

- Command flow control

- Device interface

- Windowing capability

- Graphics capability

Other utility applications have also been developed as integral parts of the software package. These include: PostScript graphics, graphical monitoring, file management, background printing, and device control.

\section{Constants and Variables}

There are five types of constants and variables: integer, long integer, float, double, and string, as shown in Table 4.1. Integer constants range from $-32,768(-215)$ to 32,767 $\left(2^{15}-1\right)$, while long integers range from $-2,147,483,648\left(-2^{31}\right)$ to $2,147,483,647\left(2^{31}-1\right)$. Double type constants are distinguished from the float type constants by the presence of the exponent ('e') character. 
The string constants are surrounded by two double quotes ("). They are character arrays of user-definable length ( 256 by default) and are terminated by a null character (hexadecimal 0). Some special characters, such as the double quote (") and carriage return, can be embedded in a string constant when prefaced by the back-single-quote ( ) character. These special characters are listed in Appendix $C$. String constants can contain any ASCII codes $(0 \times 00-0 \times F F)$ preceded by the tilde $(\sim)$ character. Examples are shown in Ex. 4.2.

Explicit declaration of variables is optional, and the types of variables that appear without prior declarations are determined as follows: variables that start with characters ' $i$ ' through ' $n$ ' are long integers; all others are doubles by default. The declaration of variables can be placed anywhere, not only at the beginning of the shell or scripts.

Variables should be preceded by a '\#' character, and assignment of values to variables is done through the equal $\Leftrightarrow$ sign. Arrays of variables can be declared by specifying the dimensions in square brackets (I D). The contents of variables may be stored either in RAM (random access memory) or in the virtual memory file on disk to save main memory (i.e. RAM) space, especially when allocating arrays of large size. The default location is specified in the configuration file. To override the default, either " $/ \mathrm{v"}$ (for virtual memory) or " $/ \mathrm{m}^{n}$ (for main memory) may be specified in the allocation command. Binary data files can be loaded into variables by including a " $/ 1$ " option in the allocation command as shown in Ex. 4.1.

Ex. 4.1: Declaration and initialization of variables.

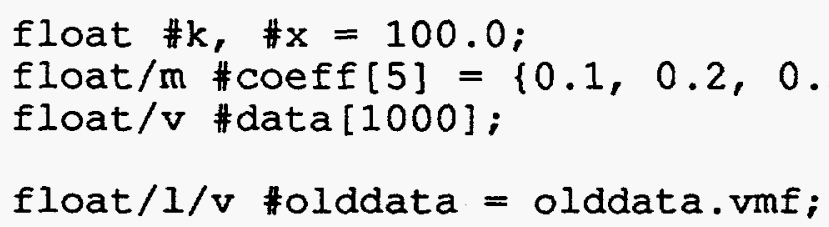

\begin{tabular}{|l|c|l|}
\hline Type & Size (bytes) & \multicolumn{1}{|c|}{ Examples } \\
\hline Integer & 2 & $0,1,-10,32767,-32768$ \\
Long integer & 4 & $1,-10,2147483647$ \\
Float & 4 & $1.0,3.5,-100.2$ \\
Double & 8 & $1 \mathrm{e}+20,-3.4 \mathrm{e}-15$ \\
String & user-defined & "This is a string.", "' "ABC'"'", "A'tB'tC" \\
\hline
\end{tabular}

Table 4.1: Valid constant types in GPDAS. 
Ex. 4.2: String constants.

write line to screen " 07 "; sound a beep.

write line to screen " $\mathrm{C} 4 \sim \mathrm{C} 4 \sim \mathrm{C} 4 \sim \mathrm{C} 4 \sim \mathrm{C} 4 \sim \mathrm{C} 4 \sim \mathrm{C} 4 \sim \mathrm{C} 4 \sim \mathrm{C} 4 \sim \mathrm{C} 4 \sim \mathrm{C} 4$ ";

! draw a line.

\section{System Global Variables}

System global variables are declared at the beginning of the shell startup and become accessible to the shell and all scripts. Currently, there are 10 such variables, as listed in Appendix C.

Ex. 5.1: Use of the system global variables.

gpib read file from hpna to \#\$vmdir 11 hpdat.vmf;

float $/ \mathrm{l} / \mathrm{v}$ \#pdat = hpdat.vmf;

\section{Alias Definition}

Commands and parts thereof may be defined as single-word aliases to simplify the interactive command input and script programming. When a predefined alias is encountered in a command string, the interpreter expands the alias and substitutes the arguments, if there are any. No space is allowed in the alias in the "define" command. In case of multiple commands, the aliases must be surrounded by curly brackets $(\{\})$, which are stripped away before being stored in the alias file.

Ex. 6.1: Defining aliases.

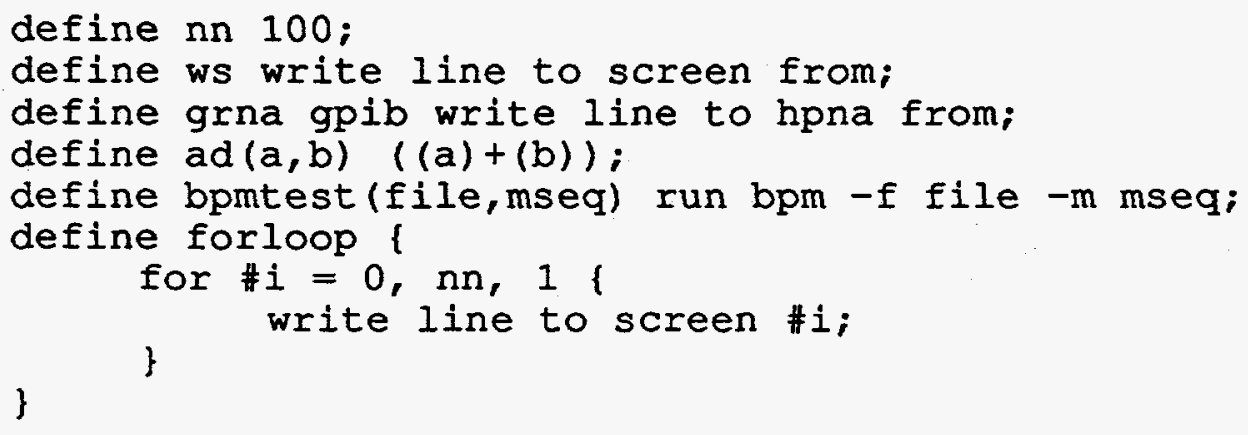

Command aliases can also be assigned to the functions keys (F1 - F10) by using the "defkey" command. Pressing one of the functions keys will display the command string and prompt for execution or further modification. 
Ex. 6.2. Function key assignment.

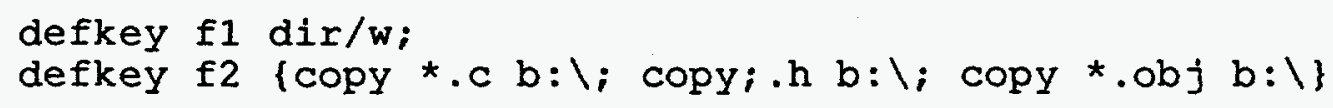

\section{History Mechanism}

All user commands entered at prompts are recorded in series in a history file for future retrieval and navigation through the commands. These commands are retrieved by using the up key $(\uparrow)$, the down key $(\downarrow)$, and the exclamation mark (!). Pressing the up key displays the command previous to the current one, and pressing the down key displays the command after the current one in a cyclic manner. The exclamation mark (!) is used to to recall the n-th command recorded in the history file.

Ex. 7.1: Retrieving a past command.

$$
\text { !10<cr> ! will display the 10-th command since startup. }
$$

\section{Show and Save}

Variables, aliases, and history can be displayed and saved to files for later use with the "show" and "save" commands. Wild characters "*1 and '?' are used to specify several variables, aliases, and previous commands. Data can be saved to binary files using the "/b" option. When saving variables, it is important that the name of the variable to be saved is at the end of the command.

Ex. 8.1: $\quad$ Showing and saving variables, aliases and history.

int \#i, \#k;

double \#x[100], \#y[100], \#cx[100], \#cy[100];

show variable \#k, \#x[*];

save variable to var.scr \#k \#x;

save /b variable to cx.vmf \#cx;

save /b variable to cy.vmf \#cy;

show variable \#c*; ! show variables starting with \#c.

save variable to varc.scr \#c*;! save variables starting

show define do*; ! show aliases starting with do.

save define to aliases.scr *; ! save all aliases.

show history 1?; ! show commands numbered between 10 and

! 19. 


\section{Input and Output}

The native operating system, such as MS-DOS, provides input/output (I/O) capabilities to peripheral devices and the built-in device drivers provide I/O capabilities to external devices as explained in Section 15 . In addition to these, several commands can be used to create, open, read from, and write to disk files and the console. The relevant commands are: "open", "close", "read", "write", and "file". The command "file" has two subcommands: "find" and "go". The syntax for these commands are tabulated in Appendix D.

Ex. 9.1: $\quad$ I/O to disk file and the console.

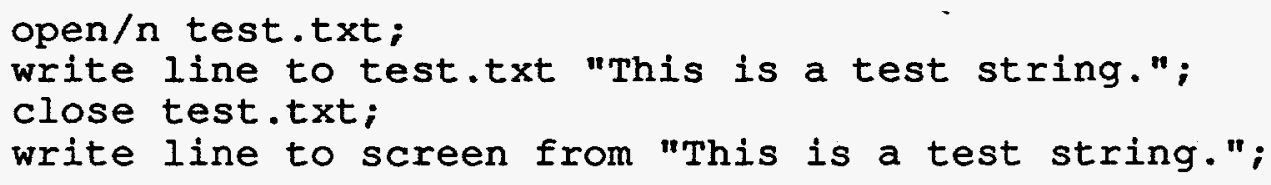

\section{Operators and Functions}

There are several operators and functions that are built into the shell. These operate on numerical and logical entities and strings. The list of built-in operators and functions is given in Appendix $\mathrm{E}$.

Ex. 10.1: Operators and functions.

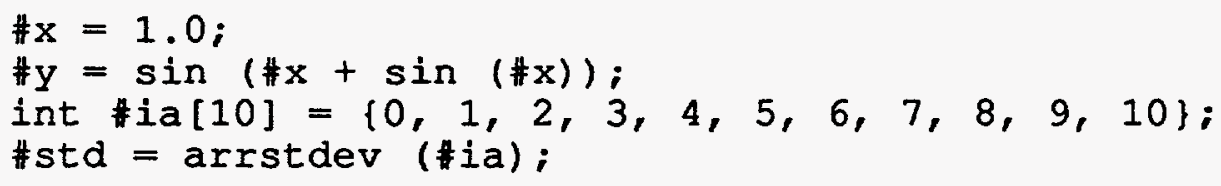

\section{Subroutines}

Subroutines are separated from the main procedure of the script by the "subroutine" heading, and the "call" command invokes the execution of a subroutine. A subroutine begins with a left curly bracket $(D$ and ends with a right curly bracket $O$ ). Since the aliases and variables are used globally among the main procedure and the subroutines, no arguments are passed between the calling routine and the one called. The "return" command simply makes the script execution resume at the point where 
the subroutine was called. If there is no return command, the subroutine returns when the end bracket is encountered.

Ex. 11.1: Calling a subroutine.

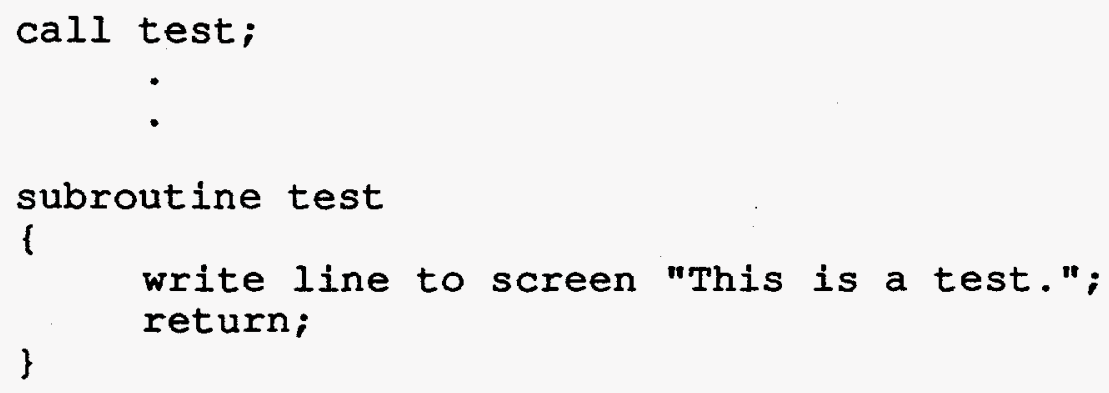

12. Command Flow Control

There are seven commands for command flow control (if, elseif, else, do, for, while, and goto). These commands must be immediately followed by a curly bracket $(D$ and a matching bracket $(0)$ at the end. They can also be nested within each other to an indefinite degree.

If, elseif, and else are conditional flow control commands. If the expression in the parentheses is non-zero, the commands surrounded by the curly brackets are executed; otherwise, the following command is executed.

Ex. 12.1: Conditional flow control commands: if, elseif and else

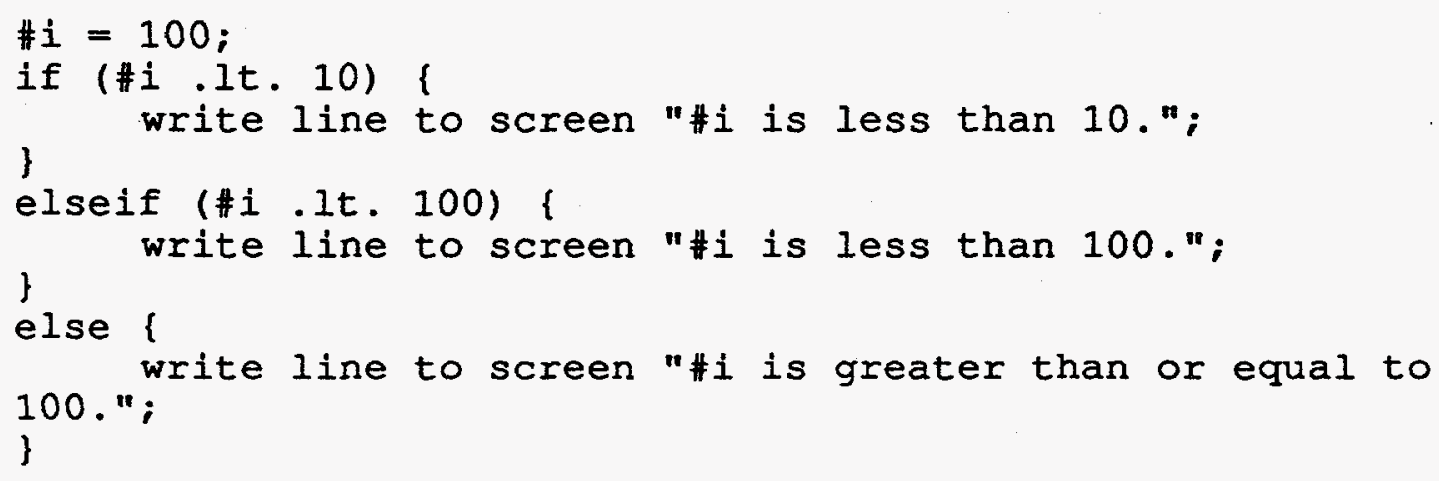

For an unconditional jump to a labeled location in a script, the "goto" command can be used. A label is identified by a colon (:) after the label name. Script execution cannot jump across nested loops using the "goto" command. 
Ex. 12.2: Unconditional flow control command: goto

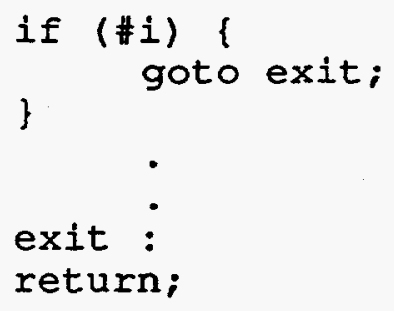

Other useful flow control commands are: do, while, and for. The "do" command is matched by a "while" condition statement at the end of the loop. After the commands within the curly brackets are executed, the "while" condition is tested. If it is true, the script execution restarts at the beginning of the loop. Otherwise, it continues with the next command. Therefore, the "do" loop is executed at least once. For the "while" command, the condition is tested first, and the execution continues with the loop or skips the entire loop depending on the result. In case of the "for" command, the initial and final values and the increment are given at the beginning of the loop. When the value of the variable goes outside the range specified by the initial and final values, the loop execution terminates.

Ex. 12.3: Flow control commands: do, while and for

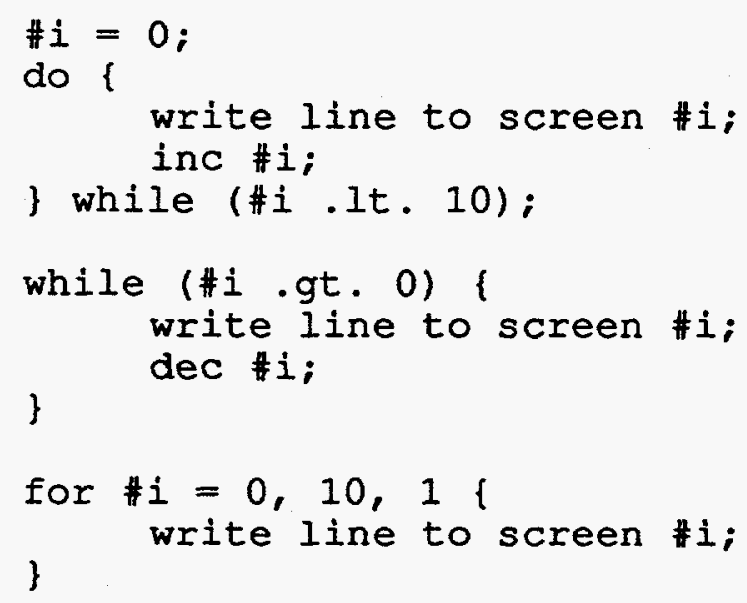

\section{Graphics}

In order to accomplish simple graphical tasks, a set of graphics commands was included in the shell. Once the shell is put in the graphics mode, the "graf" command can draw various kinds of graphical entities on the screen. This command is ignored if 
the shell is in the text mode. The "graf" command is followed by various subcommands. The syntax for the graphics commands is listed in Appendix F.

Ex. 13.1: Graphics-related commands.

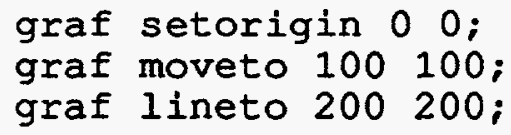

\section{Windows}

Text strings, including all printable ASCII characters, can be drawn on a window using the window-related commands. The colors of the window background and the text can be assigned for each output. The "create" subcommand creates a window but does not display it on the screen. The window is displayed with the "show" subcommand. Each window has a unique title which must be specified for each command to direct the text output to the proper destination window. The syntax for the window-related commands is listed in Appendix G.

Ex. 14.1: Window-related commands.

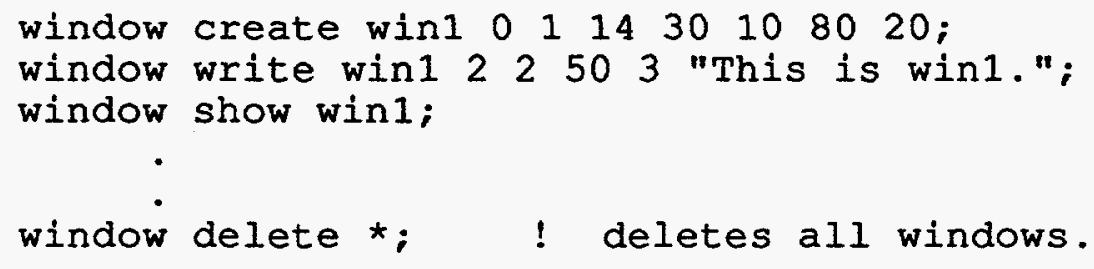

\section{GPIB and Device Drivers}

For communication with external devices, there are four drivers built in the shell: GPIB, ATMIO, ATDIO and NEAT. The corresponding commands are "gpib", "atmio", "atdio", and "neat", respectively. GPIB (General Purpose Interface Bus) is a popular parallel bus architecture for data acquisition, ATMIO is a multifunction board with A/D and D/A converters with analog $\mathrm{I} / \mathrm{O}$, and ATDIO provides high-speed 32-bit parallel digital I/O interface. These boards are manufactured by National Instruments. NEAT is a stepper motor driver board manufactured by New England Affiliated Technologies. 
The driver commands are followed by various subcommands, such as "read" and "write", as tabulated in Appendices H and I. Device drivers may be added to or deleted from the shell with minor modifications to the initialization routines in the source code.

Even though the device drivers are integral parts of the shell, each has a separate and independent command syntax, as defined by its programmer. In some cases, it is desirable not to evaluate the variables used in the device command, e.g, when reading a string from an external device and storing it in a string variable. By putting an ampersand (\&) in front of the variable, the interpreter does not evaluate the variable when parsing the command.

The "gpib" command is used for $1 / O$ and device control for external GPIB devices. Several I/O options allow downloading and uploading data either to a string variable ("line") or to a file ("file" and "ffile"). The command syntax and the functions of subcommands are tabulated in Appendix $\mathrm{H}$.

\section{Ex. 15.1: GPIB commands.}

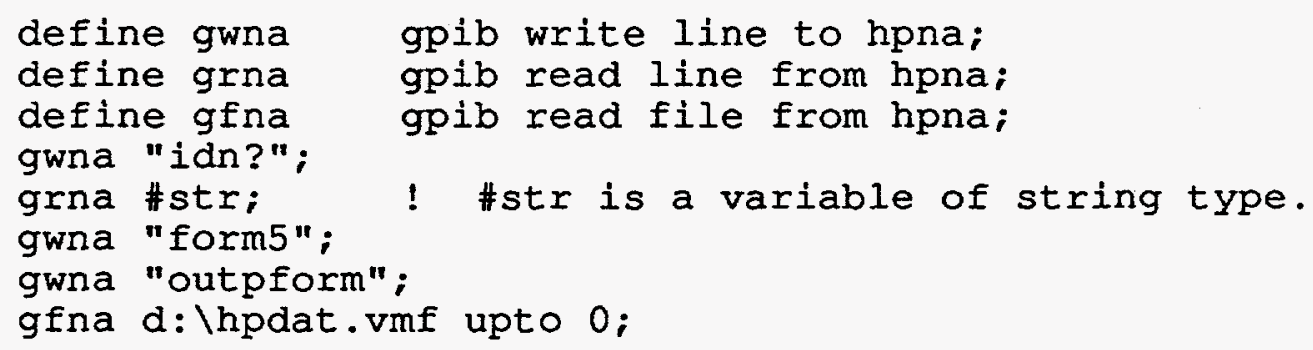

\section{Help Facility}

Extensive on-line help is available to reference the command syntax and other features of GPDAS. The help information, contained in a text file with a user-definable name ("GPDAS.HLP" by default), must conform to the following format. A main topic, usually a command, is preceded by a dollar sign (\$). A subtopic is then preceded by a double dollar sign (\$), a subsubtopic is preceded by a triple dollar sign $(\$ \$$ ), and so on. This facilitates updating the on-line help information.

Ex. 16.1: Help file format.

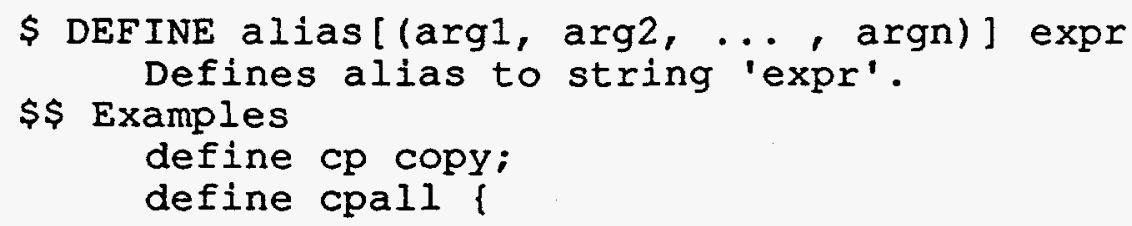




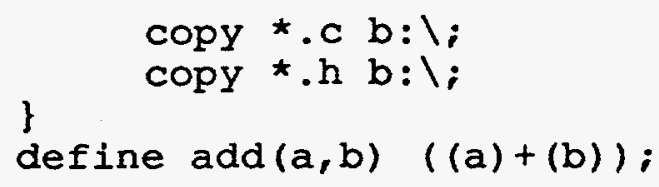

From Ex. 16.1, the command "help define" will display everything from "DEFINE" up to the double dollar sign followed by a list of available subtopics. Typing in "help gpib abort" will display everything from "abort" up to the triple dollar sign, followed by a list of available subsubtopics.

Listings of sample scripts SORTCAP.SCR and RADTRIG.SCR are in Appendices J and $\mathrm{K}$, respectively. 


\section{Appendix A. GPDAS.CFG (GPDAS Configuration File)}

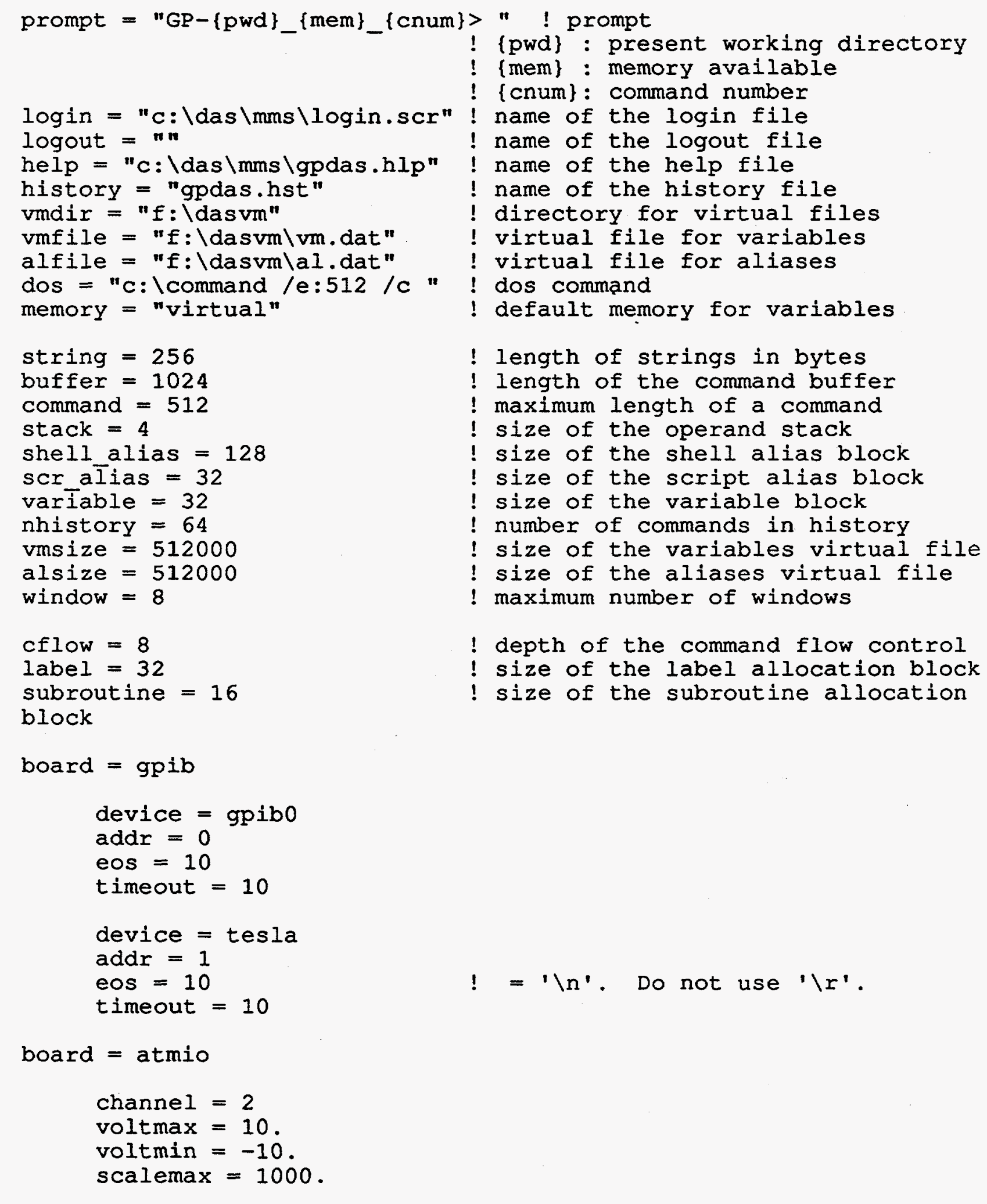


scalemin $=900$.

channel $=3$

voltmax $=10$.

voltmin $=-10$.

scalemax $=1000$.

scalemin $=500$.

board = atdio

device $=$ PSCON

$\operatorname{addr}=0 \times 14$

device $=$ ADCON

$\operatorname{add} r=0 \times 18$ 


\section{Appendix B. GPDAS Shell Command Syntax}

\begin{tabular}{|c|c|c|}
\hline Commands & Syntax & Function \\
\hline$?$ & ? command & $\begin{array}{l}\text { Substitute all applicable aliases and evaluate all } \\
\text { expressions, but do not execute the } \\
\text { command. }\end{array}$ \\
\hline : & label : & $\begin{array}{l}\text { Designates a label position for use with 'goto' } \\
\text { command. }\end{array}$ \\
\hline$=$ & variable[index] = value & Sets the value of a variable[index]. \\
\hline add & add variable [by] expr & add 'expr' to 'variable'. \\
\hline atdio & atdio subcommand ... & atdio device command. See Appendix I. \\
\hline atmio & atmio subcommand ... & atmio device command. See Appendix I. \\
\hline bkp & bkp & Set a breakpoint in a script file for debugging. \\
\hline break & break & $\begin{array}{l}\text { Break out of the loop while executing the } \\
\text { script. }\end{array}$ \\
\hline call & call subroutine & Call a subroutine in the current script. \\
\hline close & close filename & Close a file already open for $\mathrm{I} / \mathrm{O}$. \\
\hline command & command expr & $\begin{array}{l}\text { Invokes the GPDAS command interpreter. } \\
\text { 'expr' is evaluated before execution. }\end{array}$ \\
\hline $\begin{array}{l}\text { continue } \\
\text { countl }\end{array}$ & $\begin{array}{l}\text { continue } \\
\text { countl [on I off] }\end{array}$ & $\begin{array}{l}\text { Go to the beginning of the loop and continue. } \\
\text { Turn line counting on or off. If the line } \\
\text { counting is on, screen output will pause after } \\
\text { each screenful of output. }\end{array}$ \\
\hline deassign & $\begin{array}{l}\text { deassign [define I } \\
\text { defkey I variable] arg1, } \\
\arg 2, \ldots \text {, argn }\end{array}$ & $\begin{array}{l}\text { Remove currently allocated aliases or } \\
\text { variables. }\end{array}$ \\
\hline debug & debug [on I off] & $\begin{array}{l}\text { Turn on or off the feature of debugging the } \\
\text { internal stack operation. }\end{array}$ \\
\hline dec & dec variable & Decrease the value of a 'variable' by 1 . \\
\hline define & $\begin{array}{l}\text { define alias[(arg1, arg2, } \\
\ldots, \text {, argn)] expr }\end{array}$ & $\begin{array}{l}\text { Defines alias to string 'expr'. 'expr' may be } \\
\text { multiple commands, in which case 'expr' } \\
\text { must be surrounded by curly brackets (I }) \text {. }\end{array}$ \\
\hline defkey & defkey fkey expr & $\begin{array}{l}\text { Assign a command to a function key (F1 - } \\
\text { F10). }\end{array}$ \\
\hline
\end{tabular}




\begin{tabular}{|c|c|c|}
\hline Commands & Syntax & Function \\
\hline div & div variable [by] expr & Divide 'variable' with 'expr'. \\
\hline do & do $\{\ldots\}$ while (expr) & $\begin{array}{l}\text { Execute the commands in }\{\ldots\} \text { while 'expr' is } \\
\text { non-zero. }\end{array}$ \\
\hline dos & dos expr & $\begin{array}{l}\text { Invokes the DOS command interpreter. } \\
\text { 'expr' is evaluated before execution. }\end{array}$ \\
\hline double & $\begin{array}{l}\text { double } V / \mathrm{v} / \mathrm{m}] \text { variable } \\
\text { [dimen] }[=\{\ldots\}] \\
\text { double } /[/ \mathrm{v} / \mathrm{m}] \text { variable } \\
\text { = virtual_filename }\end{array}$ & $\begin{array}{l}\text { Allocate double variables. All variables are } \\
\text { initialized to } 0 \text { unless specified. If the variable } \\
\text { is an array, only the unspecified elements will } \\
\text { be initialized to } 0 .\end{array}$ \\
\hline dup & dup [on I off] & $\begin{array}{l}\text { Turn the duplication mode on/off. The } \\
\text { duplicated command shows the interpreted } \\
\text { command on the screen. }\end{array}$ \\
\hline if, elseif, else & $\begin{array}{l}\text { if }(\operatorname{expr} 1)\{. .\} \\
\text { elseif }(\operatorname{expr} 2)\{. .\} \\
\text { else }(\operatorname{expr} 3)\{. .\}\end{array}$ & $\begin{array}{l}\text { Conditional command flow control. The } \\
\text { commands in }\{\ldots\} \text { will be executed if the } \\
\text { conditional statement is true. }\end{array}$ \\
\hline end & end & $\begin{array}{l}\text { Terminate the execution of the script and } \\
\text { return to the calling script or the shell. }\end{array}$ \\
\hline file & file subcommand & $\begin{array}{l}\text { Operate on file position pointers. See } \\
\text { Appendix D. }\end{array}$ \\
\hline float & $\begin{array}{l}\text { float }[/ \mathrm{v} / \mathrm{m}] \text { variable } \\
{[\text { dimen] }[=[\ldots]]} \\
\text { float } / \mathrm{V} / \mathrm{v} / \mathrm{m}] \text { variable }= \\
\text { virtual_filename }\end{array}$ & $\begin{array}{l}\text { Allocate float variables. All variables are } \\
\text { initialized to } 0 \text { unless specified. If the variable } \\
\text { is an array, only the unspecified elements will } \\
\text { be initialized to } 0 .\end{array}$ \\
\hline for & $\begin{array}{l}\text { for var }=\text { init_expr, } \\
\text { fin_expr, inc_expr }\{\ldots\}\end{array}$ & $\begin{array}{l}\text { Execute the commands in }(\ldots\} \text { at an } \\
\text { incremental step of 'inc_expr' for 'var', } \\
\text { starting with 'init_expr'. }\end{array}$ \\
\hline goto & goto label & $\begin{array}{l}\text { Unconditional command flow control. Will } \\
\text { jump to the specified label unconditionally. }\end{array}$ \\
\hline $\begin{array}{l}\text { gpib } \\
\text { graf }\end{array}$ & $\begin{array}{l}\text { gpib subcommand } \\
\text { graf subcommand }\end{array}$ & $\begin{array}{l}\text { Invokes the GPIB function. See Appendix } \mathrm{H} \text {. } \\
\text { Invokes several graphics-related commands. } \\
\text { See Appendix F. }\end{array}$ \\
\hline help & $\begin{array}{l}\text { help [command_name } \\
\text { I function_name] }\end{array}$ & $\begin{array}{l}\text { Display help information about commands or } \\
\text { built-in functions. }\end{array}$ \\
\hline inc & inc variable & Increase the value of 'variable' by 1 \\
\hline
\end{tabular}




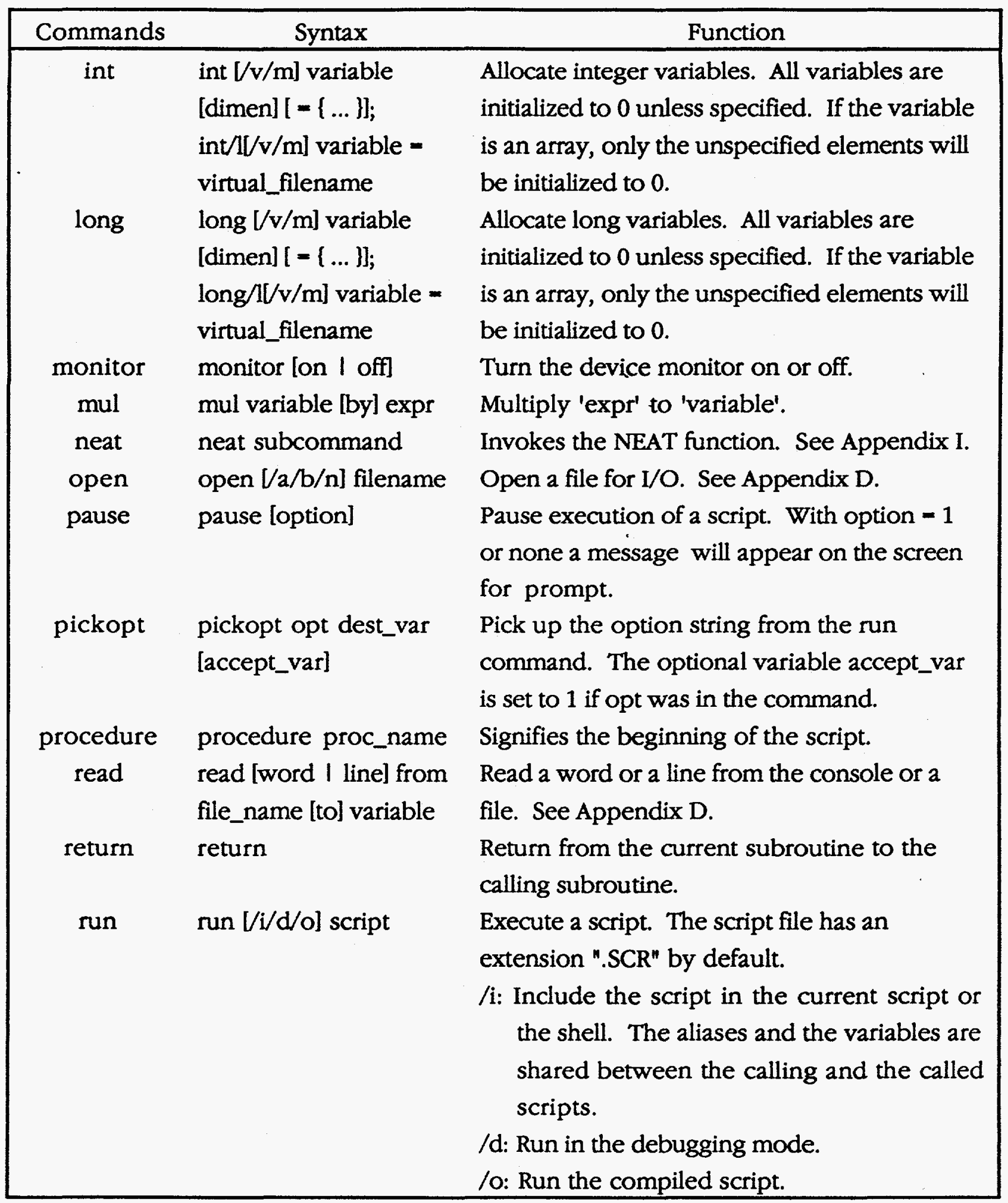




\begin{tabular}{|c|c|c|}
\hline Commands & Syntax & Function \\
\hline save & $\begin{array}{l}\text { save }[/ \mathrm{a} / \mathrm{b} / \mathrm{c} / \mathrm{s} / \mathrm{t} \text { ] [define } \\
\text { | history } \mid \text { variable] [to] } \\
\text { dest srce }\end{array}$ & $\begin{array}{l}\text { Save the currently defined aliases, history, or } \\
\text { variables ('srce') to the file 'dest'. } \\
\text { Options: } \\
\text { /a: The output will be appended to an existing } \\
\text { file. If the file does not exist, a new one } \\
\text { will be created. } \\
\text { /b: The output will be in binary form. } \\
\text { Applies when saving variables. } \\
\text { /c: No linefeed character will be added after } \\
\text { each element when saving variables. } \\
\text { /s: Displays only the names of aliases, } \\
\text { variables, and function keys assigned. } \\
\text { /t: The output will be in text form. Applies } \\
\text { when saving variables. }\end{array}$ \\
\hline show & $\begin{array}{l}\text { show } \text { V/s] [define I } \\
\text { defkey I variable] [v] }\end{array}$ & $\begin{array}{l}\text { Shows the content of ' } v \text { '. } \\
\text { Option: } \\
\text { /s: Displays only the names of aliases, } \\
\text { variables, and function keys assigned. }\end{array}$ \\
\hline string & $\begin{array}{l}\text { string }[\mathrm{v} / \mathrm{m}] \text { variable } \\
{[\text { dimen] }[=\{\ldots\}]} \\
\text { string } /[/ \mathrm{v} / \mathrm{m}] \text { variable }= \\
\text { virtual_filename }\end{array}$ & $\begin{array}{l}\text { Allocate string variables. All variables are } \\
\text { initialized to null unless specified. If the } \\
\text { variable is an array, only the unspecified } \\
\text { elements will be initialized to null. }\end{array}$ \\
\hline $\begin{array}{l}\text { sub } \\
\text { subroutine } \\
\text { video }\end{array}$ & $\begin{array}{l}\text { sub variable [by] expr } \\
\text { subroutine sub_name } \\
\text { video [graphics I text] }\end{array}$ & $\begin{array}{l}\text { Subtract 'expr' from 'variable'. } \\
\text { Signifies the beginning of a subroutine. } \\
\text { Switch between the graphics and the text } \\
\text { mode for screen output. }\end{array}$ \\
\hline wait & $\begin{array}{l}\text { wait luntil I for I tol } \\
\text { time }\end{array}$ & $\begin{array}{l}\text { Pause the execution of a script and wait } \\
\text { Options: } \\
\text { until: will wait until the specified datetime. } \\
\text { 'time' is in the form "h:m:s } \mathrm{m} / \mathrm{d} / \mathrm{y}^{\mathrm{m}} \text {. } \\
\text { for: wait for the specified time in seconds } \\
\text { starting now. } \\
\text { to: wait for the specified time in seconds, } \\
\text { starting from the beginning of the script. }\end{array}$ \\
\hline
\end{tabular}




\begin{tabular}{|cll|}
\hline Commands & \multicolumn{1}{c|}{ Syntax } & \multicolumn{1}{c|}{ Function } \\
\hline while & while (expr) $\{\ldots\}$ & $\begin{array}{l}\text { Execute the commands in }\{\ldots\} \text { while 'expr' is } \\
\text { non-zero. } \\
\text { window }\end{array}$ \\
& window subcommand \\
Invokes several window-related commands. \\
write & $\begin{array}{l}\text { write [char I word I } \\
\text { line I file] [to] dest } \\
\text { [from] srce }\end{array}$ & $\begin{array}{l}\text { Output the string in srce to 'dest', which is the } \\
\text { destination file name. See Appendix D. }\end{array}$ \\
\end{tabular}

Table B.1: List of the GPDAS shell commands. 
Appendix C. Special Characters and System Global Variables

\begin{tabular}{|c|c|}
\hline Character & Contents \\
\hline 0 & Null character (NULL) \\
\hline b & Backspace (BS) \\
\hline$-\mathrm{f}$ & Form feed (FF). \\
\hline 'n & Carriage return (CR). \\
\hline ir & Line feed (LF). \\
\hline$\cdot t$ & Horizontal tab (TAB) \\
\hline$' x$ & same as $\mathrm{x} . \mathrm{x}$ is any character other than above. \\
\hline
\end{tabular}

Table C.1: List of special characters.

\begin{tabular}{|c|c|c|}
\hline Name & Type & Contents \\
\hline$\#$ \#alfile & string & Name of the file containing aliases definitions. \\
\hline \#\$helpfile & string & Name of the help file. \\
\hline$\#$ \$historyfile & string & Name of the history file. \\
\hline \#\$loginfile & string & Name of the login file. \\
\hline \#\$logoutfile & string & Name of the logout file. \\
\hline \#\$maxstrsize & integer & Size of string variables and constants. \\
\hline$\#$ \$maxwindow & integer & Maximum number of windows that can be created. \\
\hline \#\$memvirtual & integer & $\begin{array}{l}\text { Default location of variables and aliases. } 1 \text { if disk, } 0 \\
\text { if RAM. }\end{array}$ \\
\hline$\#$ \$vmdir & string & $\begin{array}{l}\text { Directory name holding virtual memory files with } \\
\text { extensions ".VMX", }(X=I, L, F, D, S) \text {. }\end{array}$ \\
\hline \#\$vmfile & string & Name of the file containing virtual variables. \\
\hline
\end{tabular}

Table C.2: List of system global variables. 
Appendix D. V/O Command Syntax

\begin{tabular}{|c|c|c|}
\hline Commands & Syntax & Function \\
\hline open & open $[/ \mathrm{a} / \mathrm{b} / \mathrm{n}]$ filename & $\begin{array}{l}\text { Open a file for } \mathrm{I} / \mathrm{O} \text {. } \\
\text { Options: } \\
\text { /a: append mode. The subsequent } \mathrm{I} / \mathrm{O} \text { will be } \\
\text { directed to the end of the file. } \\
\text { /b: binary mode. The file will open in binary } \\
\text { mode. } \\
\text { /n: new file. If a file of the same name already } \\
\text { exists, it will be deleted and a new file will } \\
\text { be created. }\end{array}$ \\
\hline read & $\begin{array}{l}\text { read [word I line] from } \\
\text { file_name [to] variable }\end{array}$ & $\begin{array}{l}\text { Read a word or a line from the console or a } \\
\text { file. 'file_name' can be "screen" or the name } \\
\text { of a file or the string variable for file name. It } \\
\text { is important that the name of the variable is at } \\
\text { the end of the command. }\end{array}$ \\
\hline write & $\begin{array}{l}\text { write [char I word I line } \\
\text { I file] [to] dest [from] srce }\end{array}$ & $\begin{array}{l}\text { Output the string in file 'srce' to 'dest', which } \\
\text { is the destination file name. If 'dest' is } \\
\text { "screen", the output will be directed to the } \\
\text { standard I/O. Otherwise, 'dest' must be the } \\
\text { name of the file that has been already opened } \\
\text { by the 'open' command. }\end{array}$ \\
\hline file find & file find dest [in] srce & $\begin{array}{l}\text { Find string 'dest' in a file 'srce' and reposition } \\
\text { the file pointer. }\end{array}$ \\
\hline file go & $\begin{array}{l}\text { file go to [beginning I } \\
\text { current I end] [off foff] } \\
\text { [in] srce }\end{array}$ & $\begin{array}{l}\text { Go to the specified offset location in file } \\
\text { 'srce'. }\end{array}$ \\
\hline
\end{tabular}

Table D.1: The commands for $\mathrm{I} / \mathrm{O}$ to disk files and the console. The file name for the console is "screen". The characters in square brackets are optional. 
Appendix E. Operator Syntax

\begin{tabular}{|c|c|c|}
\hline Operator & Type & Function and Example \\
\hline$a+b$ & - & arithmetic addition; $\# x=\# y+2$ \\
\hline$a-b$ & - & arithmetic subtraction; $\# \mathrm{x}=\# \mathrm{y}-2$ \\
\hline$a * b$ & - & arithmetic multiplication; $\# \mathrm{x}=\# \mathrm{y} \cdot 2$ \\
\hline$a / b$ & - & arithmetic division; \#x=\#y/2 \\
\hline$a \% b$ & - & modulus (remainder after division); \#x = \#y \% 2 \\
\hline $\mathrm{a} \wedge \mathrm{b}$ & - & power; \#x = \#y $\wedge 0.5$ \\
\hline not. a & integer & logical negation; if (.not. \#i). \\
\hline a .lt. b & integer & true if $a$ is less than $b$; if (\#x.lt. 2$)$ \\
\hline a.le. $b$ & integer & true if $a$ is less than or equal to $b$; if (\#x .le. 2) \\
\hline a g.gt. b & integer & true if $a$ is greater than $b$; if (\#x .gt. 2) \\
\hline a.ge. $b$ & integer & true if $a$ is greater than or equal to $b$; if (\#x .ge. 2) \\
\hline$a$.eq. $b$ & integer & true is a is equal to b; if (\#s .eq. "this") \\
\hline$a \cdot n e \cdot b$ & integer & true is a is not equal to b; if (\#s .ne. "this") \\
\hline a .and. $b$ & integer & true if both $a$ and $b$ are true; if (\#i and. \#j) \\
\hline a .or. b & integer & true if either $a$ or $b$ is true; if (\#i .or. \#j) \\
\hline $\operatorname{int}(a)$ & integer & type cast to integer; $\# x=\operatorname{int}(2.4)$ \\
\hline long (a) & long & type cast to long integer; $\# x=$ long (2.4); \\
\hline float (a) & float & type cast to float; $\# \mathbf{x}=$ float (2); \\
\hline double (a) & double & type cast to double float; $\# \mathrm{x}=$ double (2); \\
\hline string (a) & string & type cast to string; $\# \mathrm{x}=$ string (2); \\
\hline capital (a) & string & change to upper case; if (capital (\#x) .eq. "YES") \\
\hline btoi (a) & integer & convert a binary string to integer; \#x = btoi ("0b1010"); \\
\hline itob (a) & string & convert an integer to a binary string; $\# \mathrm{x}=$ itob (10); \\
\hline htoi (a) & integer & convert a hexadecimal string to integer; $\# \mathbf{x}=$ htoi ("0x0a"); \\
\hline itoh (a) & string & convert an integer to a hexadecimal string; $\# \mathbf{x}=$ itoh (10); \\
\hline picknum $(s, n)$ & - & pick the n-th number in the string s; \#x = picknum (\#s, 2); \\
\hline pickchar $(s, n)$ & string & pick the n-th character in the string $s ; \# x=$ pickchar $(\# s, 2)$ \\
\hline pickword $(s, n)$ & string & pick the $n$-th word in the string s; \#x = pickword (\#s, 2); \\
\hline $\begin{array}{l}\text { format (s, a1,a2, } \\
\qquad . ., \text { an) }\end{array}$ & string & $\begin{array}{l}\text { format a string using the format specification 's' with } \\
\text { arguments } 21, a 2, \ldots, \text { an. }\end{array}$ \\
\hline
\end{tabular}




\begin{tabular}{|c|c|c|}
\hline Operator & Type & Function and Example \\
\hline strsize (s) & integer & length of the string $s$ in bytes; $\# l=$ strsize $(\# s)$; \\
\hline fexist (a) & integer & $\begin{array}{l}\text { true if a file of name "a" exists in the current directory; } \\
\text { if (fexist ("data.dat")) }\end{array}$ \\
\hline fsize (a) & long & the size of a file "a" in bytes.; \#flen = fsize ("data.dat"); \\
\hline diskspace (a) & long & $\begin{array}{l}\text { the available disk space in the drive a in bytes.; } \\
\text { \#dspace = diskspace ("c"); }\end{array}$ \\
\hline \multirow[t]{4}{*}{ datetime (a) } & string & $a=0$; the current time and date \\
\hline & long & $a=1$; number of seconds since startup in long integer \\
\hline & double & $a=2 ;$ number of seconds since startup in double \\
\hline & long & $a=3$; number of milliseconds since startup in long integer \\
\hline memory (a) & long & $a=0 ;$ the size of available memory in bytes \\
\hline $\min (a, b)$ & - & the smaller of $a$ and $b$; \#x $=\min (\# y, \# z)$; \\
\hline $\max (a, b)$ & - & the larger of $a$ and $b ; \# x=\max (\# y, \# z)$; \\
\hline abs (a) & - & the absolute value of $a ; \# x=a b s(-10)$; \\
\hline sqrt (a) & double & the square root of $a$; $\# x=$ sqrt $(10)$; \\
\hline $\sin (a)$ & double & the sine value of a in radian; $\# x=\sin (2.0)$; \\
\hline $\cos (a)$ & double & the cosine value of $a$ in radian; $\# x=\cos (2.0)$; \\
\hline $\tan (a)$ & double & the tangent value of a in radian; $\# x=\tan (2.0)$ \\
\hline $\operatorname{asin}(\mathrm{a})$ & double & the arc sine value of $a ; \# x=\operatorname{asin}(0.5)$ \\
\hline $\operatorname{acos}(a)$ & double & the arc cosine value of $a ; \# x=a \cos (0.5)$; \\
\hline $\operatorname{atan}(\mathrm{a})$ & double & the arc tangent value of $a ; \# x=a \tan (0.5) ;$ \\
\hline $\operatorname{atan} 2(a, b)$ & double & same as $\operatorname{atan}(\mathrm{a} / \mathrm{b}) ; \# \mathrm{x}=\operatorname{atan}(-1,2)$; \\
\hline $\log (a)$ & double & the natural logarithm of $a ; \# x=\log (2)$; \\
\hline $\exp (\mathrm{a})$ & double & the exponential of $a$; $\# x=\exp (2)$; \\
\hline rand (a) & double & the random number generator of $\max a ; \# x=$ rand (1); \\
\hline $\operatorname{sign}(\mathrm{a})$ & integer & the sign of the number $a ; \# x=\operatorname{sign}(-100)$; \\
\hline $\operatorname{arrdim}(a)$ & long & the dimension of the array $\mathrm{a} ; \# \mathrm{x}=\operatorname{arrdim}(\# \mathrm{y})$; \\
\hline $\operatorname{arrmax}(a)$ & - & the maximum of the array a; \#x = arrmax (\#y); \\
\hline $\operatorname{arrmin}(\mathrm{a})$ & - & the minimum of the array $a$; $\# x=\operatorname{arrmin}(\# y)$; \\
\hline arrmean (a) & double & the mean value of the array a; \#x = arrmean (\#y); \\
\hline arrstdev (a) & double & the standard deviation of the array $a ; \# x=$ arrstdev (\#y); \\
\hline $\operatorname{arrsum}(\mathrm{a})$ & double & the sum of the elements of the array $\mathrm{a} ; \# \mathrm{x}=\operatorname{arrsum}(\# \mathrm{y})$; \\
\hline
\end{tabular}




\begin{tabular}{|ccc|}
\hline Operator & Type & Function and Example \\
\hline arrsum2 (a) & double & the sum of the square of the elements; $\# \mathrm{x}=\operatorname{arrsum} 2(\# \mathrm{y}) ;$ \\
arrdot $(\mathrm{a}, \mathrm{b})$ & double & the dot product of the arrays a and $\mathrm{b} ; \# \mathrm{x}=\operatorname{arrdot}(\# \mathrm{y}, \# \mathrm{z}) ;$ \\
\hline
\end{tabular}

Table E.1: List of operators and functions built into the shell. The type of the return value depends upon the arguments if it is not specified. 
Appendix F. Graphics Command Syntax

\begin{tabular}{|c|c|c|}
\hline Commands & Syntax & Function \\
\hline arc & graf arc radius angle1 angle 2 & $\begin{array}{l}\text { Draw an arc with radius from } \\
\text { angle1 to angle } 2 \text {. }\end{array}$ \\
\hline clear & graf clear & Clear the graphics screen. \\
\hline lineto & graf lineto $\mathrm{h} \mathrm{v}$ & Draw a line to $(h, v)$. \\
\hline moveto & graf moveto $\mathrm{h} \mathrm{v}$ & Move to $(h, v)$. \\
\hline putpixel & graf putpixel $\mathrm{h} v$ color & Put a pixel of color at $(h, v)$. \\
\hline restore & graf restore & Restore the graphics status. \\
\hline rmoveto & graf rmoveto dh dv & Move by $(\mathrm{dh}, \mathrm{dv})$. \\
\hline rlineto & graf rlineto $\mathrm{dh} d v$ & Draw a line $(d h, d v)$. \\
\hline save & graf save & Save the graphics status. \\
\hline setbkcolor & graf setbkcolor color & Set the background color. \\
\hline setcolor & graf setcolor color & Set the color for drawing. \\
\hline setfont & graf setfont fontid & Set the font for text. \\
\hline setfontsize & graf setfontsize fontsize & Set the font size for text. \\
\hline setorigin & graf setorigin $\mathrm{h} v$ & $\begin{array}{l}\text { Set the origin of coordinate to } \\
(\mathrm{h}, \mathrm{v}) \text {. }\end{array}$ \\
\hline setlinestyle & graf setlinestyle linestyle & Set the line style. \\
\hline setlinewidth & graf setlinewidth linewidth & Set the line width. \\
\hline setscale & graf setscale scale & $\begin{array}{l}\text { Set the scale for graphics object } \\
\text { and text. }\end{array}$ \\
\hline show & graf show string & Draw a text string left adjusted. \\
\hline showcenter & graf showcenter string & Draw a text string centered. \\
\hline showright & graf showright string & Draw a text string right adjusted. \\
\hline vshowdown & graf vshowdown string & $\begin{array}{l}\text { Draw a text string top to } \\
\text { bottom. }\end{array}$ \\
\hline vshowup & graf vshowup string & $\begin{array}{l}\text { Draw a text string bottom to } \\
\text { top. }\end{array}$ \\
\hline
\end{tabular}

Table F.1: List of the graphics related commands. 


\section{Appendix G. Window Command Syntax}

\begin{tabular}{|c|c|c|}
\hline Commands & Syntax & Function \\
\hline create & $\begin{array}{l}\text { window box wtitle blink bc tc } \\
\text { l t r b } \\
\text { window create wtitle blink bc } \\
\text { tc } 1 \mathrm{t} \mathrm{r} \text { b }\end{array}$ & $\begin{array}{l}\text { Draw a rectangular box }(1, t, r, b) \\
\text { around a window. } \\
\text { Create a window. }\end{array}$ \\
\hline cwrite & $\begin{array}{l}\text { window cwrite wtitle blink bc } \\
\text { tc } 1 \mathrm{t} \mathrm{r} \text { b text }\end{array}$ & $\begin{array}{l}\text { Draw a colored text in a } \\
\text { rectangle }(1, t, r, b) \text { in a window. }\end{array}$ \\
\hline debox & window debox wtitle $1 \mathrm{t} r \mathrm{~b}$ & $\begin{array}{l}\text { Remove the rectangular box }(\mathrm{l}, \mathrm{t} \text {, } \\
\mathrm{r}, \mathrm{b}) \text { in a window. }\end{array}$ \\
\hline $\begin{array}{l}\text { delete } \\
\text { fill }\end{array}$ & $\begin{array}{l}\text { window delete [wtitle I all] } \\
\text { window fill wtitle blink bc tc } 1 \\
\text { t r b }\end{array}$ & $\begin{array}{l}\text { Delete a window or all windows. } \\
\text { Fill the rectangle }(1, t, r, b) \text { with } \\
\text { bc. tc is the color of text in the } \\
\text { rectangle. }\end{array}$ \\
\hline $\begin{array}{l}\text { hide } \\
\text { move }\end{array}$ & $\begin{array}{l}\text { window hide [wtitle I all] } \\
\text { window move wtitle } 1 \mathrm{t}\end{array}$ & $\begin{array}{l}\text { Hide a window or all windows. } \\
\text { Move a window to a new } \\
\text { location. }\end{array}$ \\
\hline $\begin{array}{l}\text { resize } \\
\text { show } \\
\text { write }\end{array}$ & $\begin{array}{l}\text { window resize wtitle } \mathrm{dw} \text { dh } \\
\text { window show wtitle } \\
\text { window write wtitle } 1 \mathrm{t} \text { r b text }\end{array}$ & $\begin{array}{l}\text { Resize the window to dw } \mathrm{x} d \mathrm{~h} \text {. } \\
\text { Show a window. } \\
\text { Draw a text in a rectangle }(1, t, r \text {, } \\
\text { b) in a window. }\end{array}$ \\
\hline
\end{tabular}

Table G.1: List of the window related commands. wtitle $=$ window title, $b c=$ background color, $\mathrm{tc}=$ text color, $\mathrm{l}=$ left, $\mathrm{t}=$ top, $\mathrm{r}=$ right, $\mathrm{b}=$ bottom. 
Appendix H. GPIB Command Syntax

\begin{tabular}{|c|c|c|}
\hline Commands & Syntax & Function \\
\hline clear & gpib clear dev & Clear a GPIB device. \\
\hline command & gpib command [board brd] $\mathrm{cmd} \mathrm{cnt}$ & $\begin{array}{l}\text { Output a GPIB command } \\
\text { to a board. }\end{array}$ \\
\hline control & gpib control [to] dev & $\begin{array}{l}\text { Pass the GPIB control to } \\
\text { a device. }\end{array}$ \\
\hline ifclear & gpib ifclear dev & $\begin{array}{l}\text { Interface clear a GPIB } \\
\text { device. }\end{array}$ \\
\hline llock & gpib llock [board brd] dev & $\begin{array}{l}\text { Disable the local mode of } \\
\text { a GPIB device. }\end{array}$ \\
\hline local & gpib local dev & Make a GPIB device local. \\
\hline open & gpib open dev [timeout tmo] & Open a GPIB device. \\
\hline read & $\begin{array}{l}\text { gpib read [line I file I ffile] [from] dev } \\
\text { [to] dest [upto eosc] }\end{array}$ & $\begin{array}{l}\text { Read a word, a line, or a } \\
\text { file from a GPIB device. }\end{array}$ \\
\hline remote & gpib remote [board brd] dev [on I off] & $\begin{array}{l}\text { Enable/disable REN } \\
\text { (remote enable) line. }\end{array}$ \\
\hline trigger & gpib trigger dev & Trigger a GPIB device. \\
\hline write & $\begin{array}{l}\text { gpib write [line I file] [to] dev [from] } \\
\text { srce [upto eosc] }\end{array}$ & $\begin{array}{l}\text { Write a word, a line, or a } \\
\text { file from a GPIB device. }\end{array}$ \\
\hline
\end{tabular}

Table H.1: The commands for communication to external devices via the GPIB interface. The characters in square brackets are optional. 


\section{Appendix I. Device Driver Command Syntax}

\begin{tabular}{|cll|}
\hline Commands & \multicolumn{1}{c|}{ Syntax } & \multicolumn{1}{c|}{ Function } \\
\hline read & atmio read [line I file] & Read A/D conversion \\
& [all I chan I chan1 chan2] var & $\begin{array}{l}\text { result(s) and store to } \\
\text { 'dest'. 'dest' is either a } \\
\end{array}$ \\
& & variable name or a file \\
& name. \\
write & atmio write line [all I dac0 I dac1] expr & Output D/A conversion \\
& & result. 'expr' is the \\
& & desired voltage. \\
& &
\end{tabular}

Table 1.1: The commands for the ATMIO device driver. The characters in square brackets are optional.

\begin{tabular}{|cll|}
\hline Commands & \multicolumn{1}{c|}{ Syntax } & \multicolumn{1}{c|}{ Function } \\
\hline read & neat read [line I file] [from] srce Ito] & Read from a NEAT board \\
& dest & and store to 'dest'. 'dest' \\
& is either a variable name \\
& & or a file name. \\
Write & neat write [line I file] [to] dest [from] & Write to a NEAT board. \\
& srce & 'srce' is test string or a \\
& file name. \\
\hline
\end{tabular}

Table I.2: The commands for the NEAT device driver. The characters in square brackets are optional. 


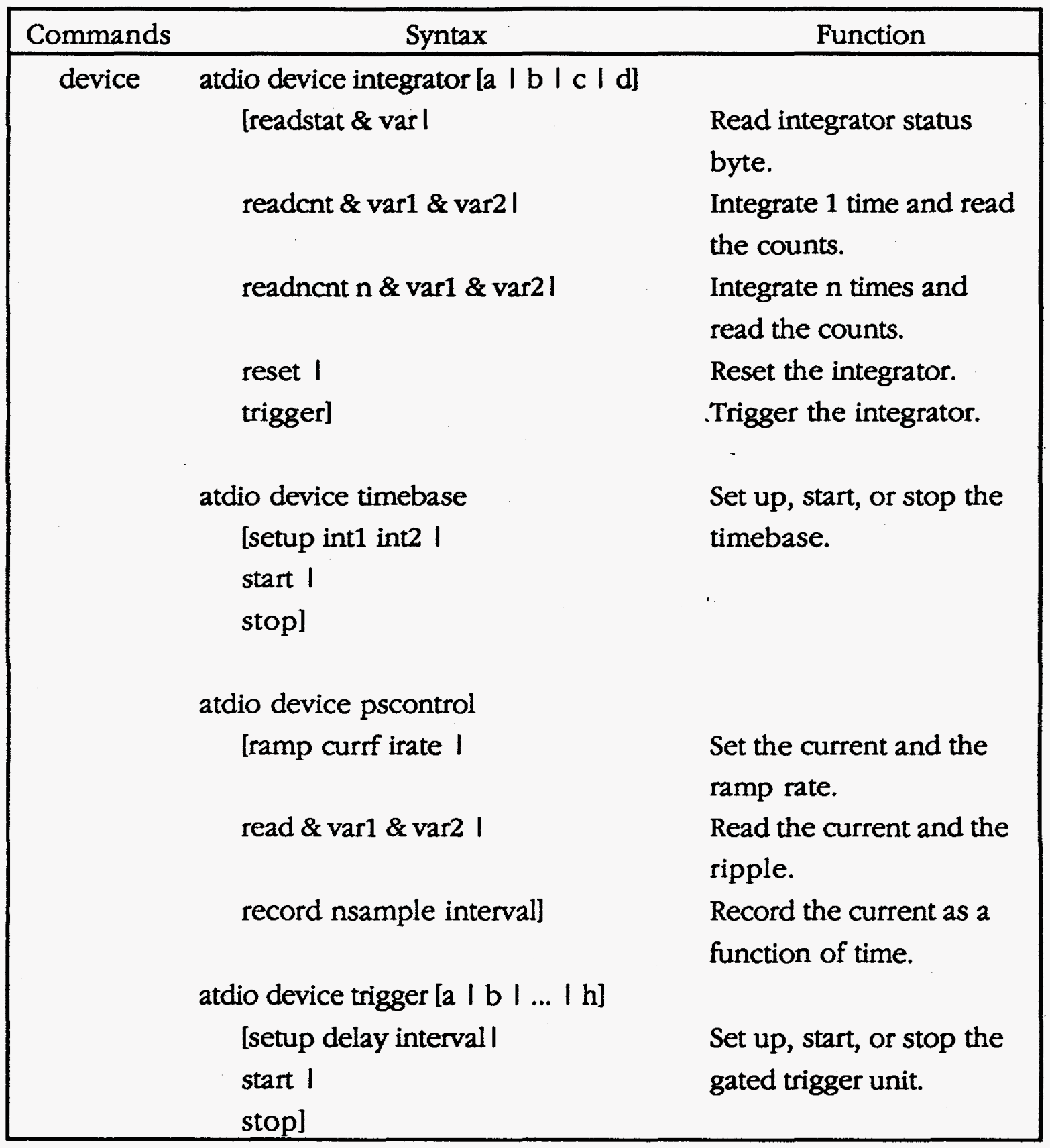




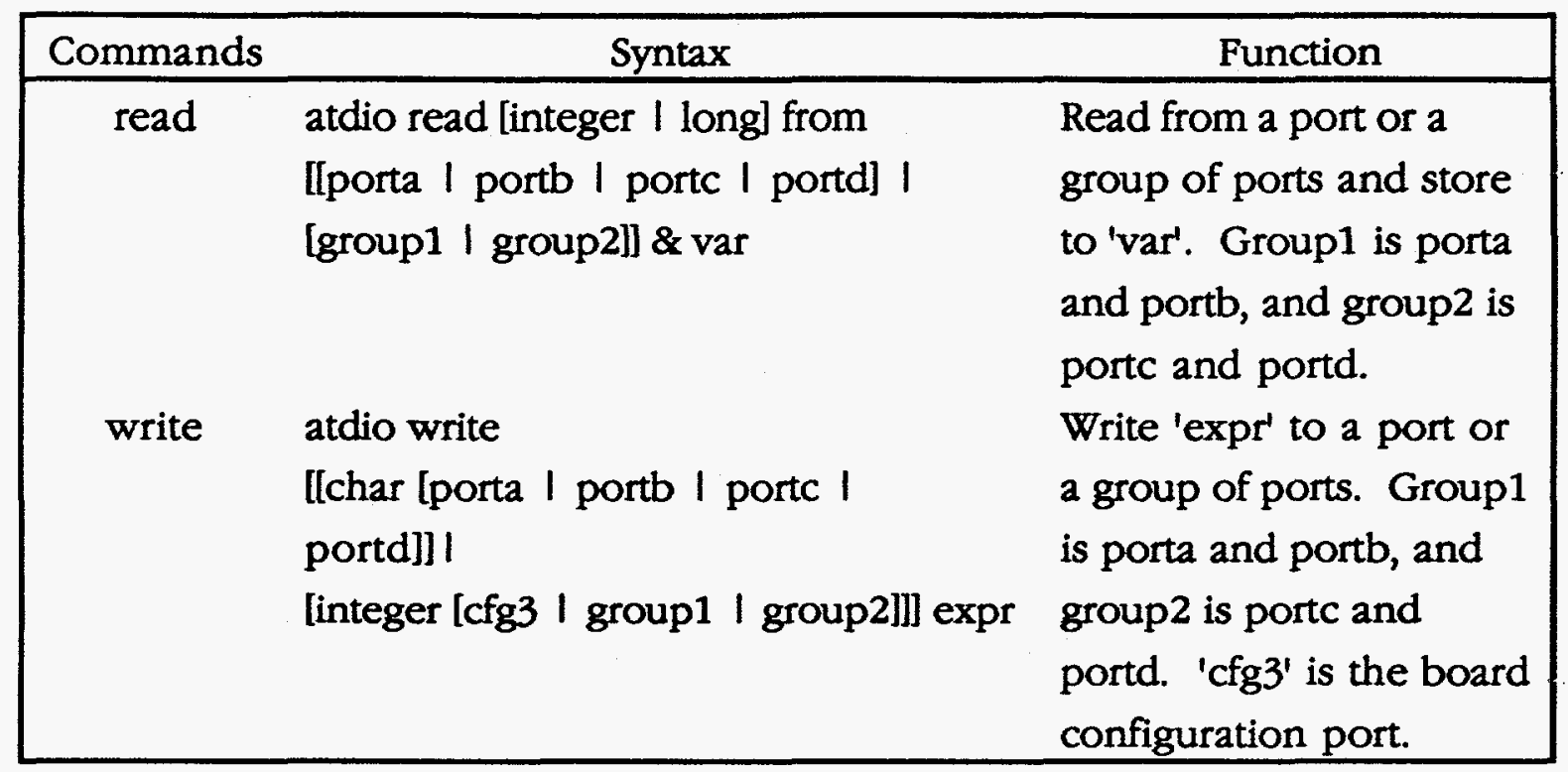

Table I.3: The commands for the ATDIO device driver. The characters in square brackets are optional. 


\section{Appendix J. Script Example SORTCAP.SCR}

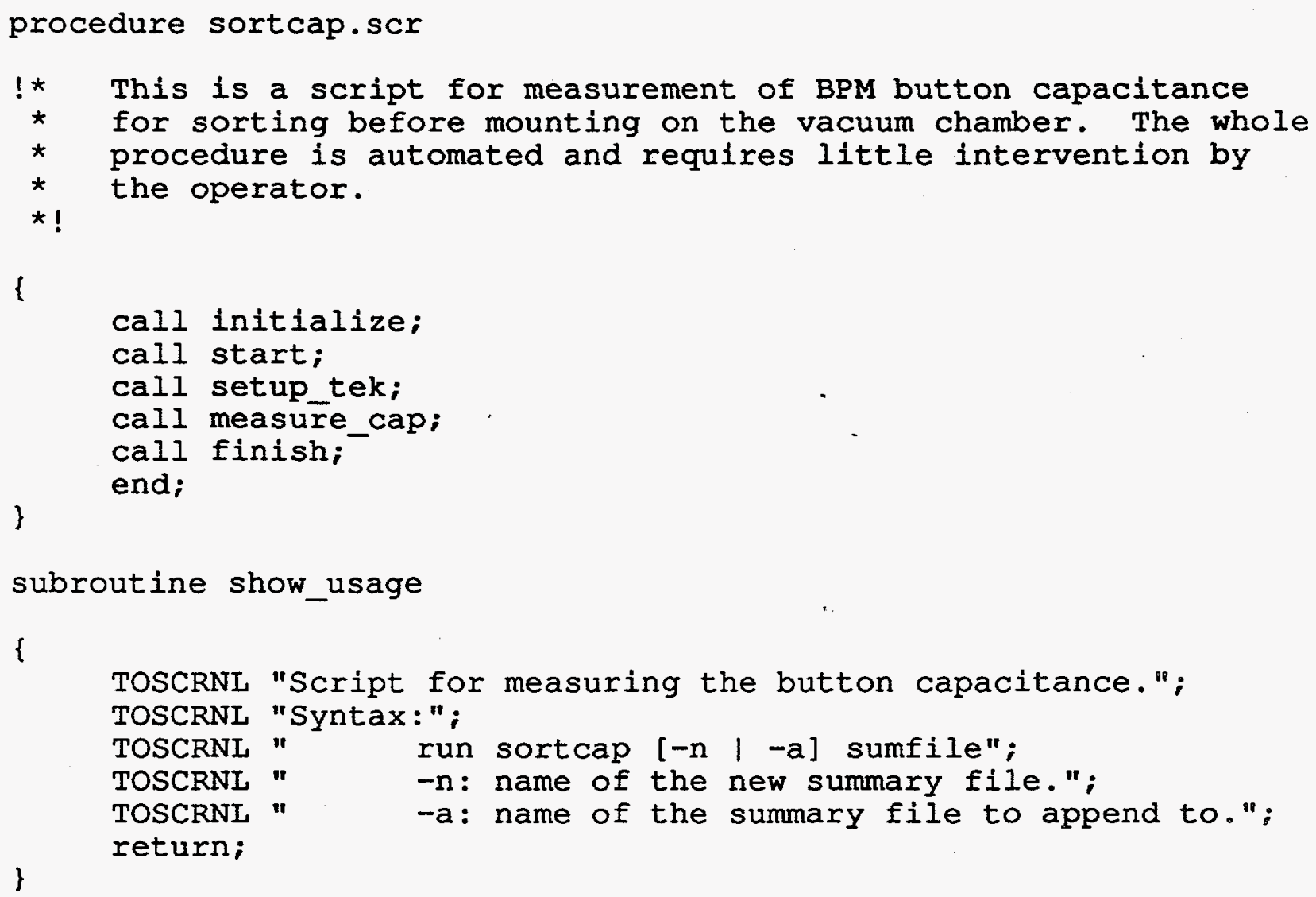


subroutine get_data

! Initialize the data display.

GWT "autos star";

GWT "chm1 offset:" || \#tekoffset || ",sensi:" || \#teksensi;

GWT "mainpos " II \#tektbase;

GWT "tbmain len:" || \#itlen || ",time:" || \#tektdiv;

GWT "navg " II \#teknavg;

GWT "tra1 des:'avg(m1)" ;

wait for tekwait;

! Get the data for normalization to obtain the saturation

! level.

GWT "curve?";

GRFTB norm. dat upto 0 ;

! Turn off averaging for subsequent data.

GWT "chm1 offset:" || \#tekoffset || ",sensi:" || \#teksensi;

GWT "tral des: 'ml'";

GWT "mainpos" \#tektbase;

GWT "tbmain time:" \#tektdiv;

wait for \#tekwait;

! Write the header data.

GWT "mainpos?";

GRT \#1ine1;

\#str_len = strsize (\#line1);

GWT "tbmain?";

GRT \#line2;

add \#str_len by strsize (\#line2) + 2 ;

TOFILEL "Header Data";

TOFILEL format ("Size = od", \#str_len);

TOFILEL \#Iine1;

TOFILEL \#Iine2;

! Write the normalization data.

TOFILEL "Normalization Data";

TOFILEL format ("Size = od", fsize (norm.dat));

TOFILEF norm.dat;

for $\# i=1$, \#nmeas, 1 l

$\# i 1=i-1 ;$

GWT "curve?";

GRFTB wave.dat upto 0 ; 


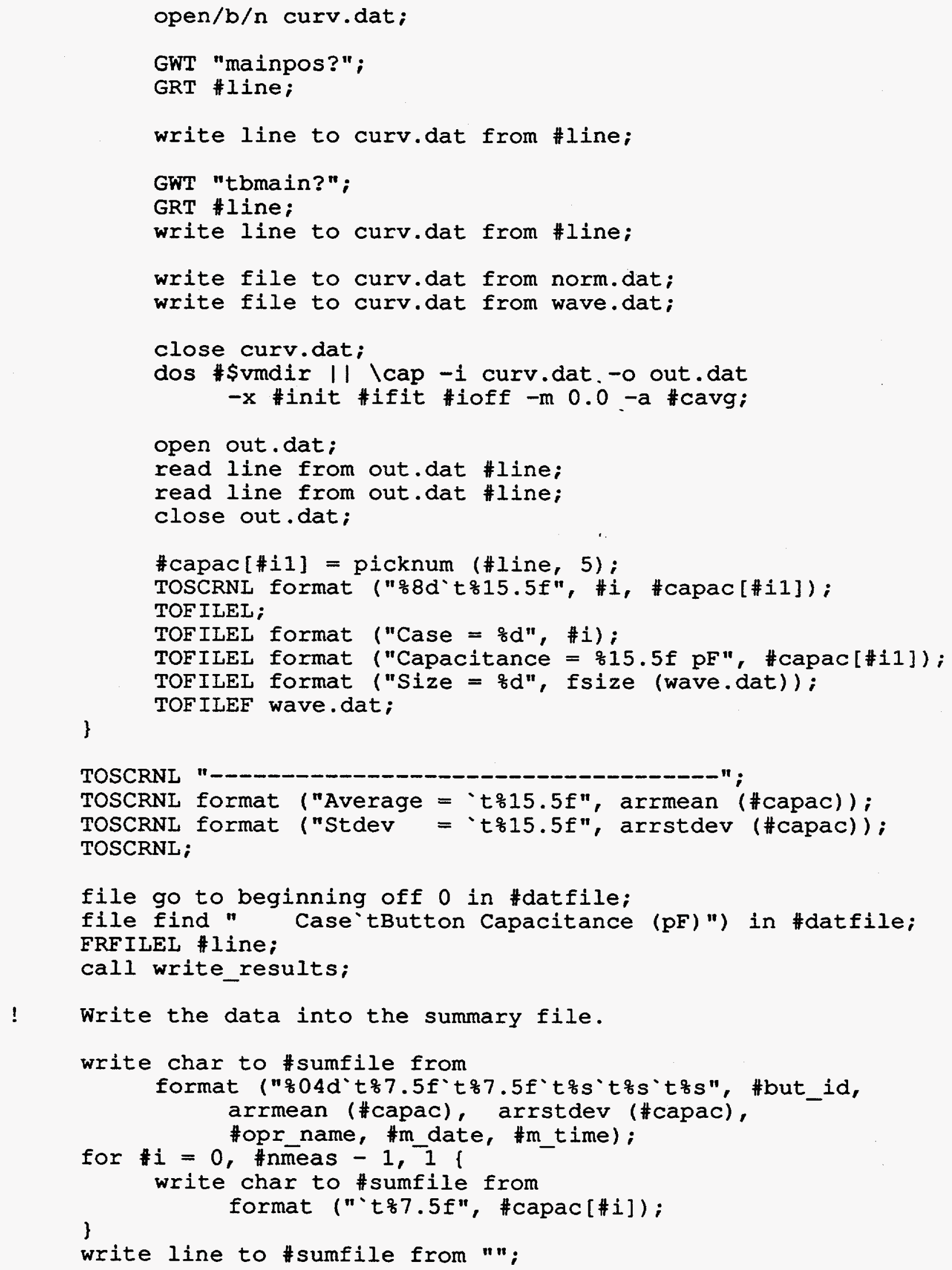




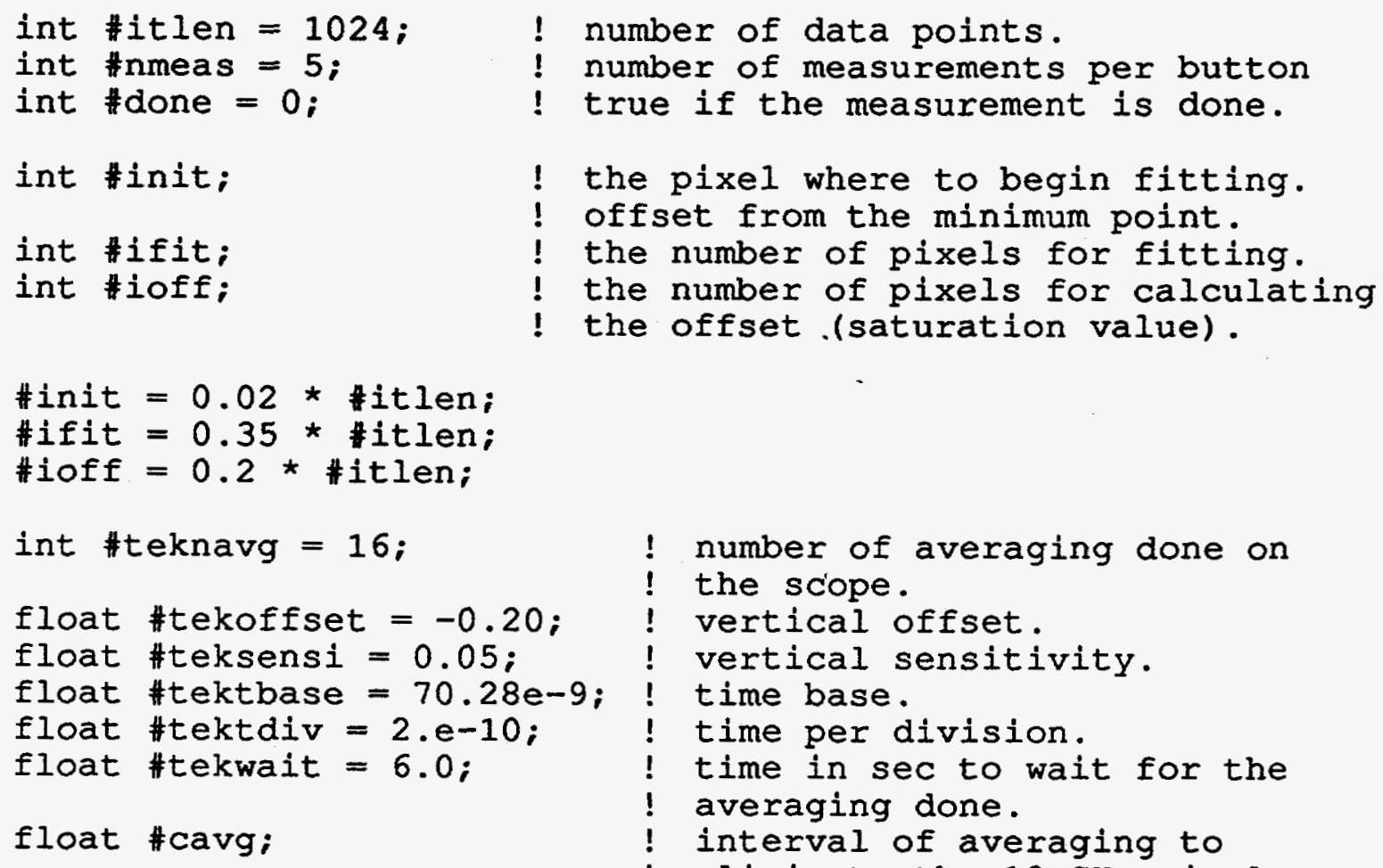


string \#m_time; ! time when the measurement was made.

! Miscellaneous variables.

int str len;

string \#Ine, \#answer, \#string;

! Aliases.

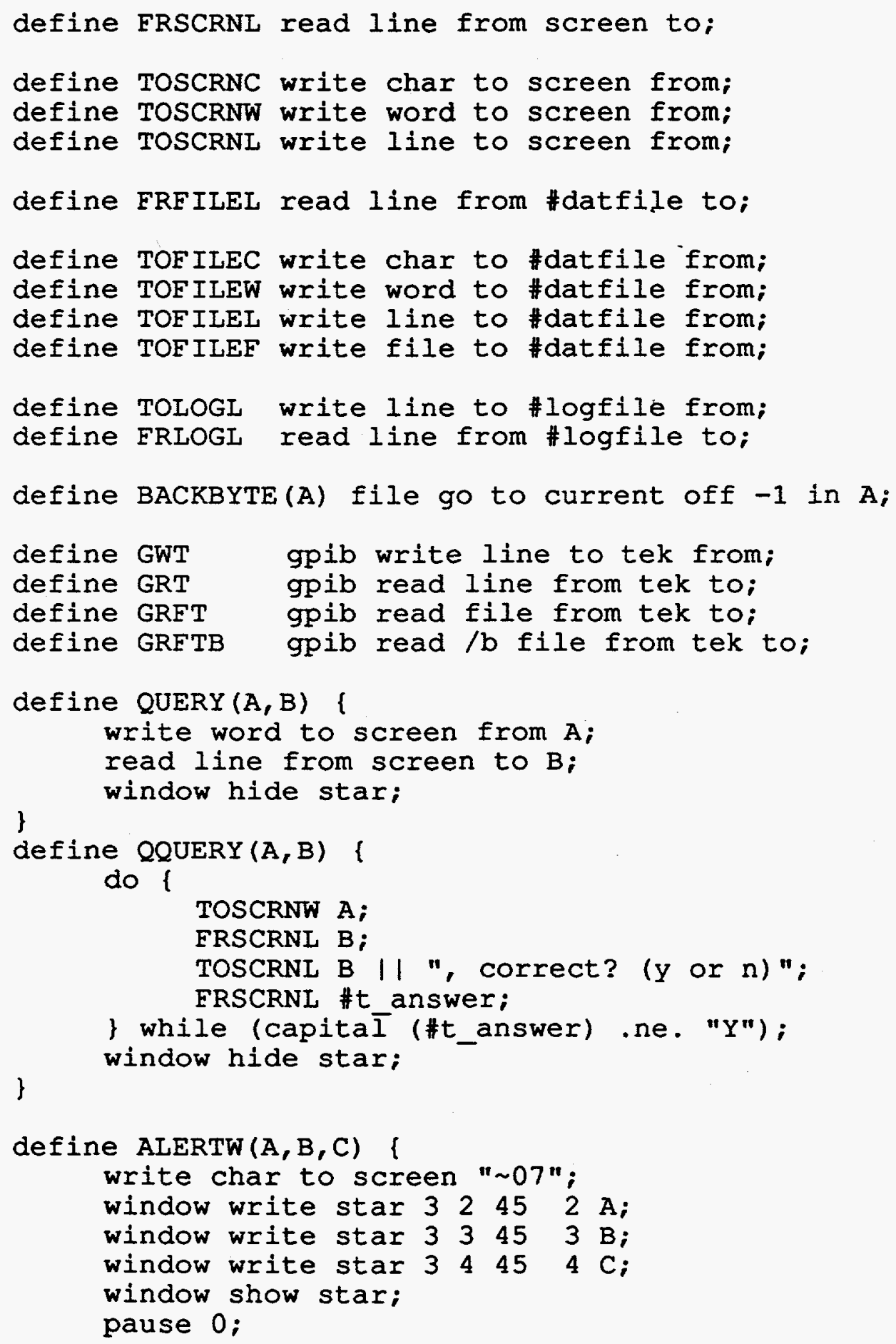




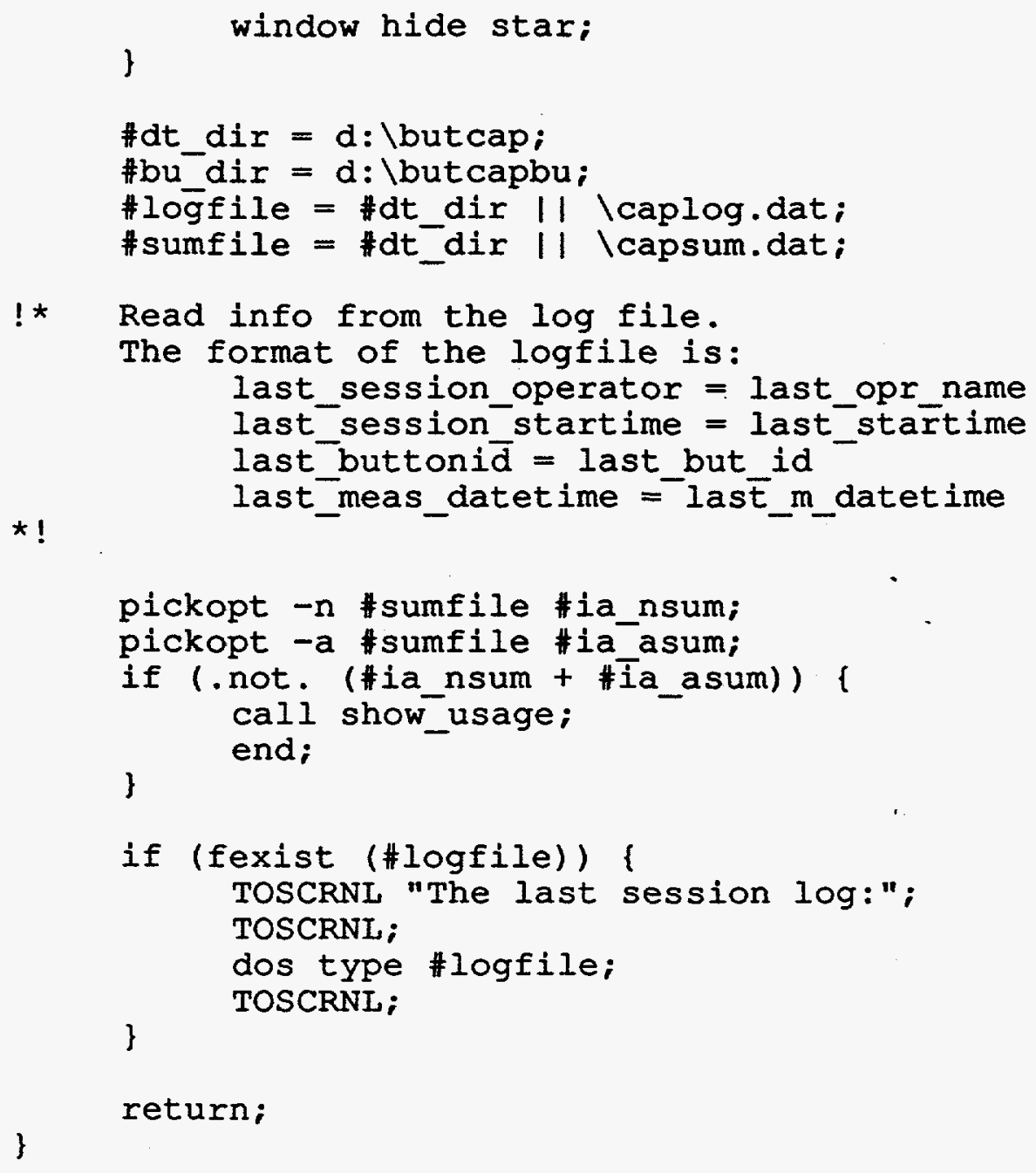




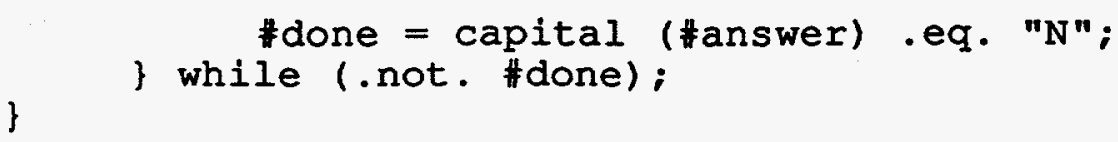

! Select the trace and the channel.

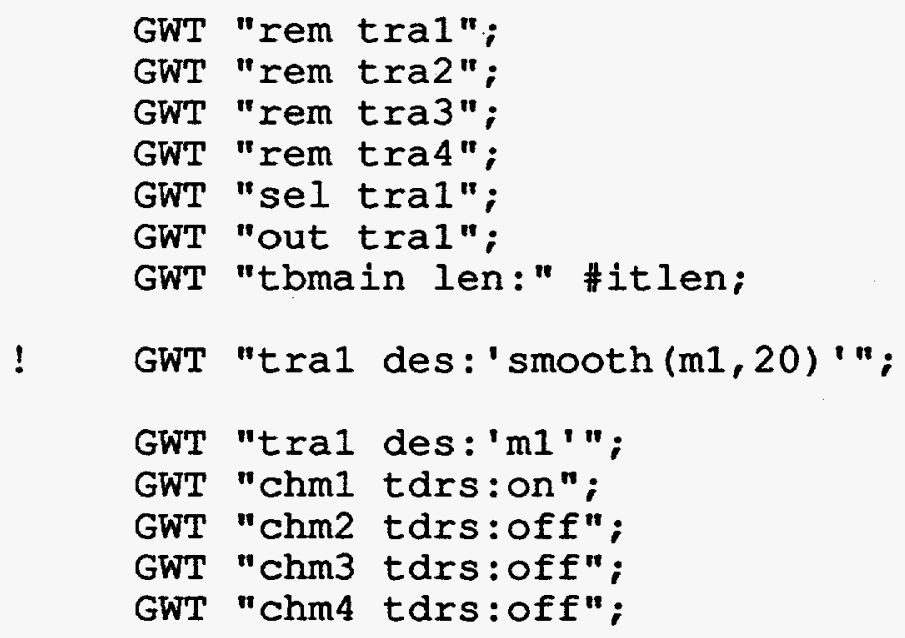

! Adjust the display offset and sensitivity.

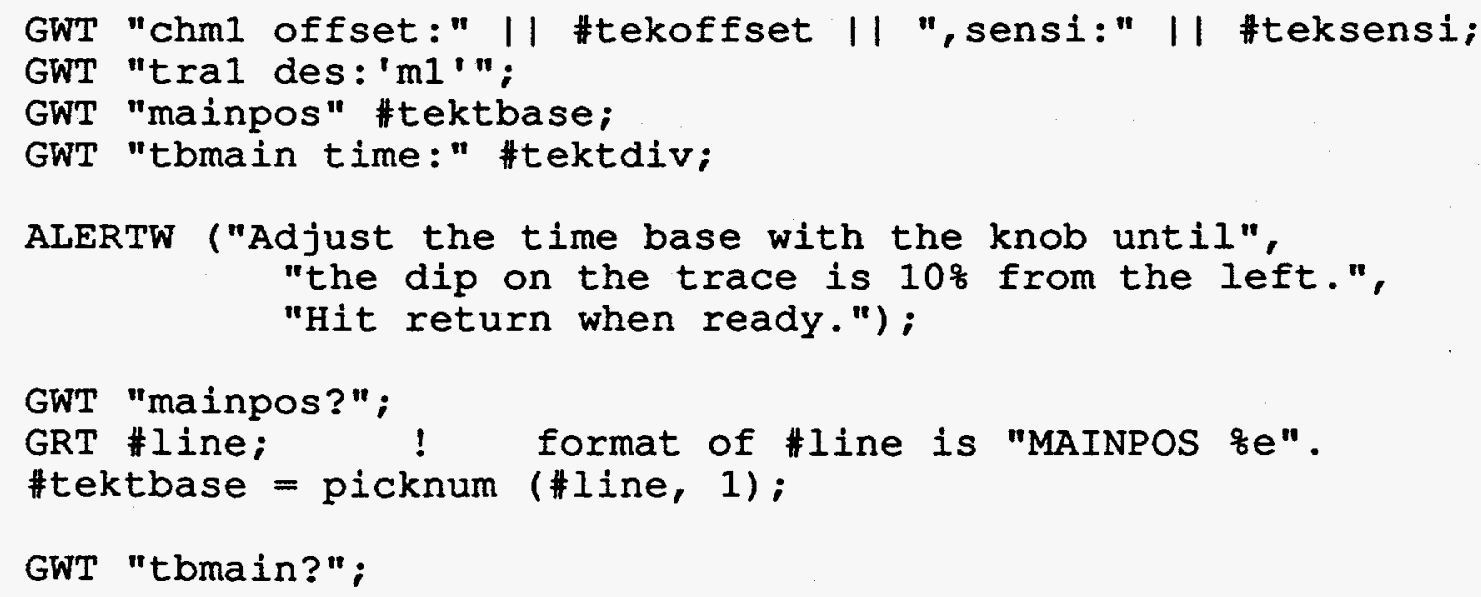




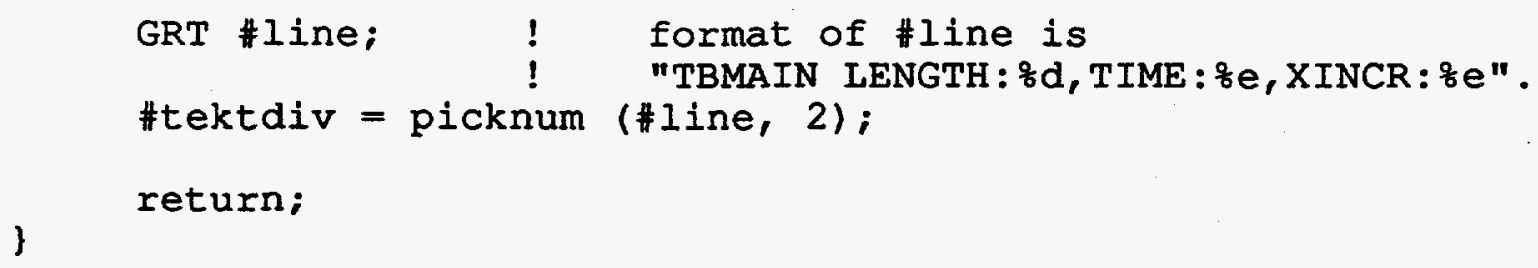




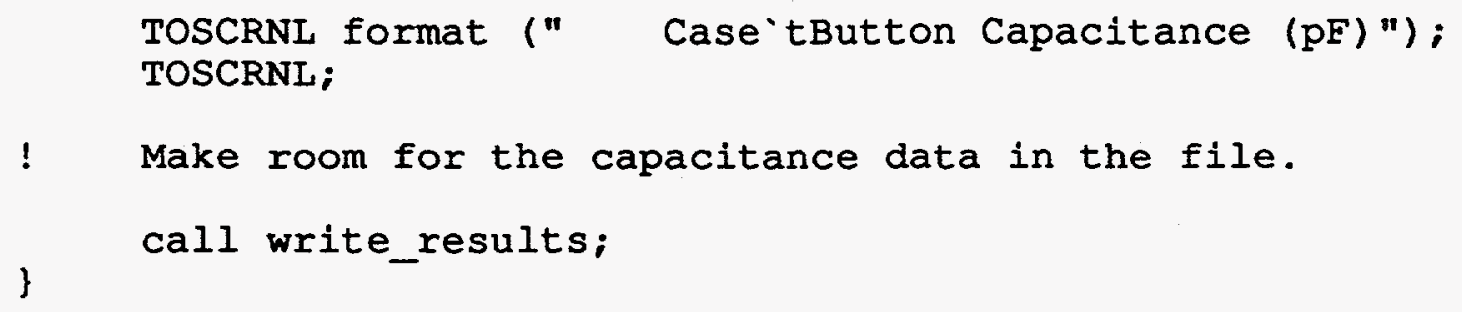




\section{Appendix K. Script Example RADTRIG.SCR}

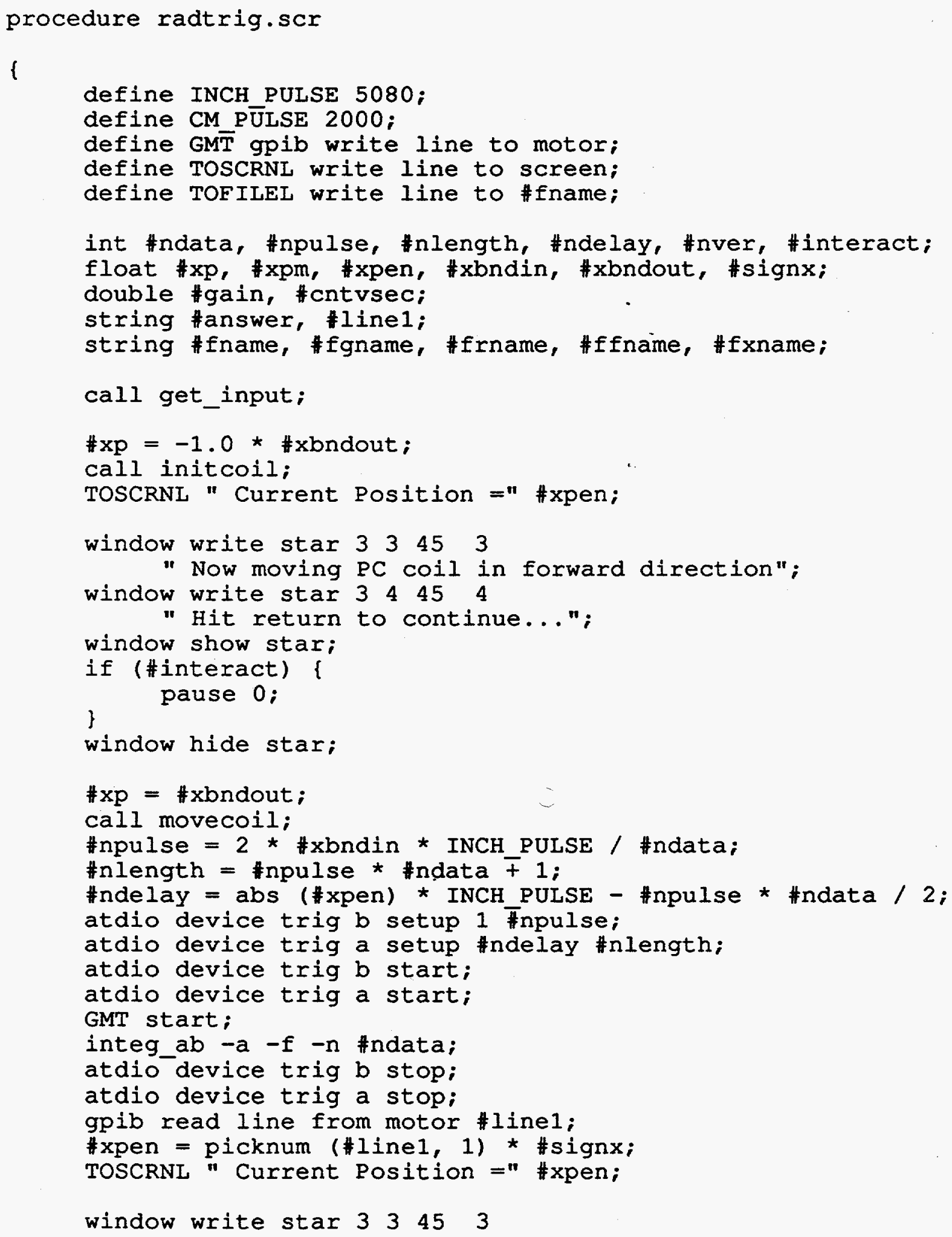


"Now moving PC coil in reverse direction";

window write star $3 \quad 4 \quad 45 \quad 4$

" Hit return to continue...";

window show star;

if (\#interact) \{

\}

pause 0 ;

window hide star;

$\# \mathrm{xp}=-1.0 *$ \#bndout;

call movecoil;

\#npulse $=2$ * \#xbndin * INCH_PULSE / \#ndata;

\#nlength $=$ npulse * \#ndata $\mp 1$;

\#ndelay = abs (\#xpen) * INCH PULSE - \#npulse * \#ndata / 2;

atdio device trig b setup 1 inpulse;

atdio device trig a setup \#ndelay \#nlength;

atdio device trig b start;

atdio device trig a start;

GMT start;

integ_ab - $a-r-n$ \#ndata;

atdio device trig b stop;

atdio device trig a stop;

gpib read line from motor \#linel;

\#xpen = picknum (\#line1, 1) * \#signx;

TOSCRNL "Current Position =" \#xpen;

call mvorig;

window write star 33453 "l;

window write star $\begin{array}{llll}3 & 4 & 45 & 4\end{array}$

" Now Doing some calculation....";

window write star $3545 \quad 5$ " ;

window show star;

float $/ 1$ \#frdt = frwd a.vmf;

float $/ 1$ \#bkdt = bkwd_a.vmf;

float \#avrg[\#ndata];

float \#avrg_add [ ndata + 1];

float \#avrg $\times x \times[$ ndata +1$]$;

\#avrg_add [0] $=0$;

\#avrg $\times x \times[0]=$ npulse $*(0.0$ - \#ndata $/ 2.0) /$ INCH_PULSE;

for $\# \bar{i}=1$, \#ndata, 1 \{

*avrg[ $i-1]=$

\#cntvsec $\star 0.5 *(\# \operatorname{frdt}[\# i-1]-\# b k d t(\# i-1])$;

\#avrg_add [*i] =

\#avrg_add [\#i - 1]+\#avrg[\#i-1];

\#avrg_xxx [ $i]=$

\} \#pulse * (\#i - \#data / 2.0) / INCH_PULSE;

save/b var to \#frname \#avrg;

save/b var to \#fxname \#avrg_xxx;

save/b var to \#ffname \#avrg_add;

window delete star;

if (\#interact) \{ 


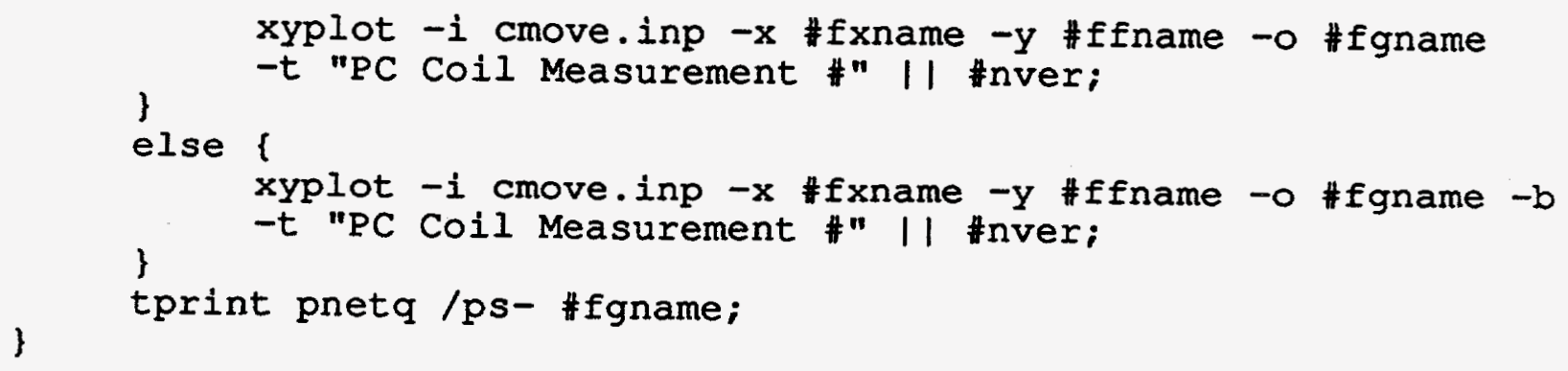




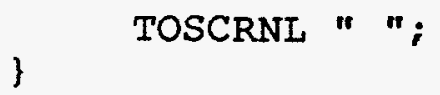




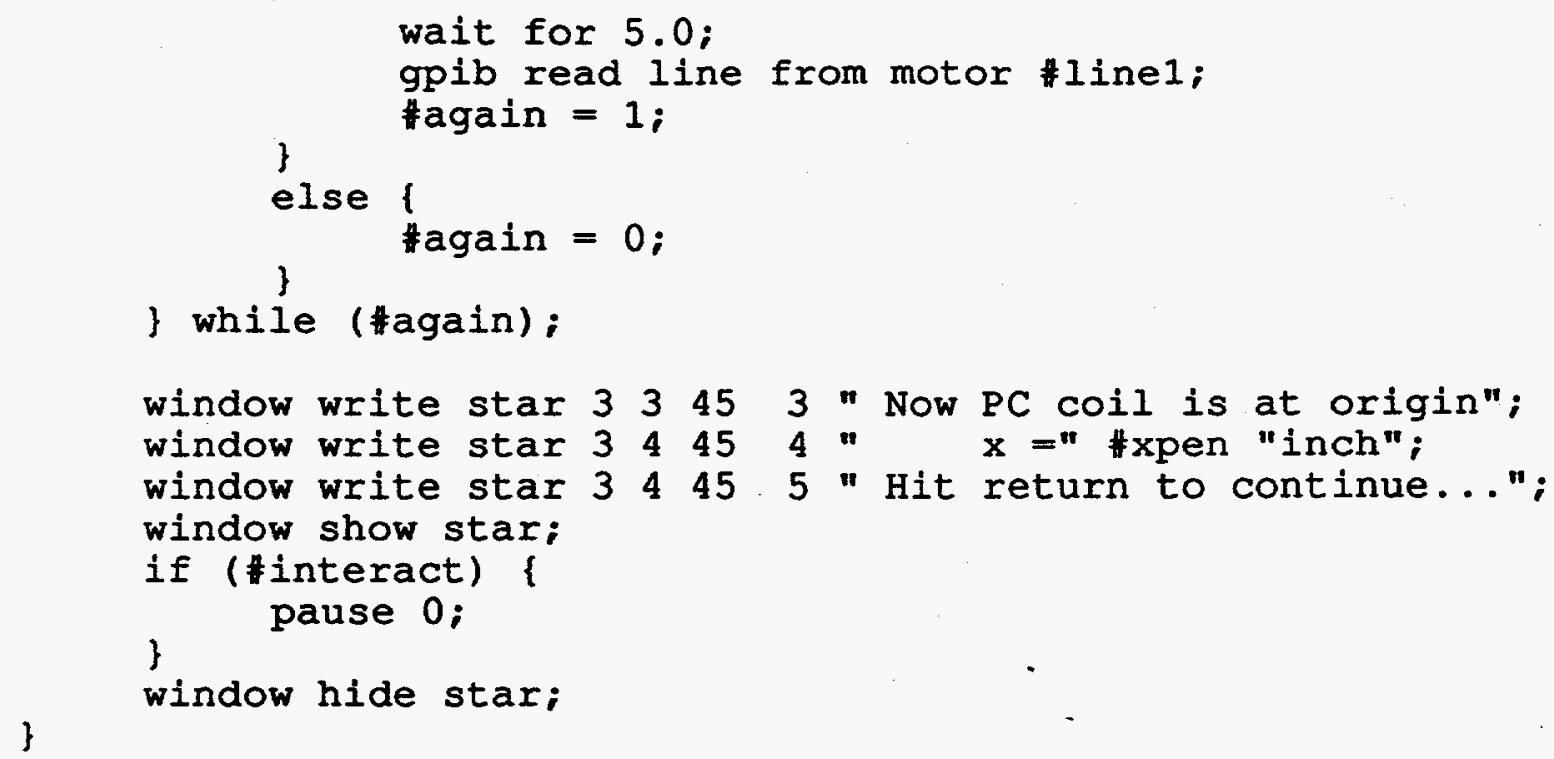

"Usage: RUN RADTRIG [-b] -v \#num [-x \#xin] [-n \#ndata]"; TOSCRNL "-b: Operate in batch mode (default : interactive)"; TOSCRNL "-v \#num : Measurement number $(99<$ \#num < 1000)"; TOSCRNL "-x \#xin : Integration boundary"; 